TRANSACTIONS OF THE

AMERICAN MATHEMATICAL SOCIETY

Volume 360, Number 12, December 2008, Pages 6331-6369

S 0002-9947(08)04571-6

Article electronically published on June 26, 2008

\title{
RIGIDITY OF GRADED REGULAR ALGEBRAS
}

\author{
E. KIRKMAN, J. KUZMANOVICH, AND J. J. ZHANG
}

\begin{abstract}
We prove a graded version of Alev-Polo's rigidity theorem: the homogenization of the universal enveloping algebra of a semisimple Lie algebra and the Rees ring of the Weyl algebras $A_{n}(k)$ cannot be isomorphic to their fixed subring under any finite group action. We also show the same result for other classes of graded regular algebras including the Sklyanin algebras.
\end{abstract}

\section{INTRODUCTION}

The invariant theory of $k\left[x_{1}, \cdots, x_{n}\right]$ is a rich subject whose study has motivated many developments in commutative algebra and algebraic geometry. One important result is the Shephard-Todd-Chevalley Theorem [Theorem 1.1 that gives necessary and sufficient conditions for the fixed subring $k\left[x_{1}, \cdots, x_{n}\right]^{G}$ under a finite subgroup $G$ of $G L_{n}(k)$ to be a polynomial ring. The study of the invariant theory of noncommutative algebras is not well understood, and it is reasonable to begin with the study of finite groups acting on rings that are seen as generalizations of polynomial rings.

We will show that in contrast to the commutative case, a noncommutative regular algebra $A$ is often rigid, meaning that $A$ is not isomorphic to any fixed subring $A^{G}$ under a nontrivial group of automorphisms $G$ of $A$. A typical result is the AlevPolo rigidity theorem that shows that both the universal enveloping algebra of a semisimple Lie algebra and the Weyl algebras $A_{n}(k)$ are rigid algebras.

Theorem 0.1 (Alev-Polo rigidity theorem $\mathrm{AP}$ ).

(a) Let $\mathfrak{g}$ and $\mathfrak{g}^{\prime}$ be two semisimple Lie algebras. Let $G$ be a finite group of algebra automorphisms of $U(\mathfrak{g})$ such that $U(\mathfrak{g})^{G} \cong U\left(\mathfrak{g}^{\prime}\right)$. Then $G$ is trivial and $\mathfrak{g} \cong \mathfrak{g}^{\prime}$.

(b) If $G$ is a finite group of algebra automorphisms of $A_{n}(k)$, then the fixed subring $A_{n}(k)^{G}$ is isomorphic to $A_{n}(k)$ only when $G$ is trivial.

The main goal of this paper is to investigate a similar question for graded algebras. As one example, in Section 6 we prove the following graded version of the Alev-Polo rigidity theorem. Let $H(\mathfrak{g})$ denote the homogenization of the universal enveloping algebra of a finite dimensional Lie algebra $\mathfrak{g}$ (the definition is given in Section 6).

Received by the editors November 6, 2006.

2000 Mathematics Subject Classification. Primary 16E10, 16W30, 20 J05.

Key words and phrases. Artin-Schelter regular algebra, group action, reflection, trace, Hilbert series, fixed subring, quantum polynomial rings. 
Theorem 0.2. $\quad$ (a) Let $\mathfrak{g}$ and $\mathfrak{g}^{\prime}$ be Lie algebras with no 1-dimensional Lie ideal. Let $G$ be a finite group of graded algebra automorphisms of $H(\mathfrak{g})$ such that $H(\mathfrak{g})^{G} \cong H\left(\mathfrak{g}^{\prime}\right)$ (as ungraded algebras). Then $G$ is trivial and $\mathfrak{g} \cong \mathfrak{g}^{\prime}$.

(b) Let $A$ be the Rees ring of the Weyl algebra $A_{n}(k)$ (with respect to the standard filtration of $A_{n}(k)$ ). Then $A$ is not isomorphic to $A^{G}$ (as ungraded algebras) for any finite nontrivial group of graded automorphisms.

Artin-Schelter regular algebras [Definition 1.5] are a class of graded algebras that are generalizations of polynomial algebras, and they have been used in many areas of mathematics and physics. One can ask whether an Artin-Schelter regular algebra $A$ can be isomorphic to a fixed subring $A^{G}$ when $G$ is a nontrivial finite group of graded algebra automorphisms of $A$. One could consider fixed rings under ungraded automorphisms ( $\mathrm{AP}$ did not restrict itself to filtered automorphisms) also, but we leave that problem to others. Although it is easy to construct noncommutative algebras $A$ and groups of automorphisms $G$ where $A^{G}$ is isomorphic to $A$ [Example 1.2, it turns out that this happens less often than we expected [Lemma [5.2(b)], and we will provide both some necessary conditions and some sufficient conditions for this problem. Our work thus far suggests that a generalization of the Shephard-Todd-Chevalley Theorem requires a new notion of a reflection group, one that depends on the Hilbert series of the Artin-Schelter regular algebra $A$ (for the conditions used in the commutative case turn out to be neither necessary nor sufficient [Example 2.3]). In this paper we focus on Artin-Schelter regular algebras that have the same Hilbert series as commutative polynomial rings. We call $A$ a quantum polynomial ring (of dimension $n$ ) if it is a noetherian, graded, ArtinSchelter regular domain of global dimension $n$, with Hilbert series $(1-t)^{-n}$. Skew polynomial rings, $H(\mathfrak{g})$, the Rees rings of the Weyl algebras, and Sklyanin algebras are all quantum polynomial rings. One of our results is the following.

Theorem 0.3 (Theorem 6.2). Let $A$ be a quantum polynomial ring. Suppose that there is no nonzero element $b \in A_{1}$ such that $b^{2}$ is normal in $A$. Then $A$ is not isomorphic to $A^{G}$ as ungraded algebras for any nontrivial finite group $G$ of graded algebra automorphisms.

If $A$ is viewed as the coordinate ring of a noncommutative affine $n$-space, then Theorem 0.3 can be interpreted as: a "very noncommutative" affine $n$-space cannot be isomorphic to any quotient space of itself under a nontrivial finite group action. If we really understood noncommutative spaces, this might be a simple fact. The hypothesis that $A$ has no normal element of the form $b^{2}$ is easy to check in many cases. For example, Theorem 0.3 applies to the non-PI Sklyanin algebras of dimension $n$.

Corollary 0.4 (Corollary 6.3). Let $S$ be a non-PI Sklyanin algebra of global dimension $n \geq 3$. Then $S$ is not isomorphic to $S^{G}$ for any nontrivial finite group $G$ of graded algebra automorphisms.

The method of proving Theorems 0.2 (a) and 0.3 is to show that $H(\mathfrak{g})^{G}$ and $A^{G}$ do not have finite global dimension for any nontrivial $G$. This method applies to other algebras such as down-up algebras (see Proposition 6.4) which are not quantum polynomial rings. However, if $A$ is the Rees ring of the Weyl algebra $A_{n}(k)$, then there are groups $G$ of automorphisms of $A$ so that $A$ has a fixed subring $A^{G}$ that is Artin-Schelter regular, but not isomorphic to $A$ [Example 5.4]. Since 
commutative polynomial rings are the only commutative (Artin-Schelter) regular algebras, the situation where $A^{G}$ is Artin-Schelter regular, but not isomorphic to $A$, does not arise in the commutative case. Hence this paper deals with a small portion of a more fundamental question: find all noetherian graded Artin-Schelter regular algebras $A$ and finite groups $G$ of graded algebra automorphisms of $A$ such that $A^{G}$ has finite global dimension. Given a well-studied quantum polynomial ring, it should be possible to find all finite groups $G$ such that $A^{G}$ has finite global dimension. Following the commutative case, we call such a group a reflection group. For algebras in Theorems 0.2 (a) and 0.3 and Corollary 0.4 , there is no nontrivial reflection group.

For the simplest noncommutative ring $k_{q}[x, y]$ with relation $x y=q y x$ for a nonzero scalar $q$ in the base field $k$, all reflection groups for $k_{q}[x, y]$ have been worked out completely, and these results motivated our approach to general ArtinSchelter regular algebras. However, the project becomes much harder when the global dimension of the algebra $A$ is higher, and less is known about large dimension Artin-Schelter regular algebras.

Some ideas in the classical Shephard-Todd-Chevalley theorem for the commutative polynomial ring can be extended to the noncommutative case. Let $A$ be a quantum polynomial ring, and let $g$ be a graded algebra automorphism of $A$. Then $g$ is called a quasi-reflection of a quantum polynomial ring of dimension $n$ if its trace is of the form

$$
\operatorname{Tr}_{A}(g, t)=\frac{1}{(1-t)^{n-1}(1-\xi t)}
$$

for some scalar $\xi \neq 1$. We classify all possible quasi-reflections of quantum polynomial rings in Theorem 3.1, which states that, with only one interesting exception, the quasi-reflections of a quantum polynomial ring are reflections of the generating space $A_{1}$ of $A$. The notion of quasi-reflection is extended to Artin-Schelter regular algebras, and we prove that for any Artin-Schelter regular algebra $A$, if $A^{G}$ has finite global dimension, then $G$ must contain a quasi-reflection [Theorem 2.4. Therefore Theorems 0.2 (a) and 0.3 follow by verifying that $H(\mathfrak{g})$ in Theorem 0.2 (a) and $A$ in Theorem 0.3 do not have any quasi-reflections. More work is required in analyzing the fixed ring of the Rees algebras of the Weyl algebras, as they have quasi-reflections and Artin-Schelter regular fixed rings [Proposition 6.7 and Corollary 6.8 .

As a secondary result we formulate a partial version of the Shephard-ToddChevalley theorem for noncommutative Artin-Schelter regular algebras.

Theorem 0.5 (Theorem 5.3). Let $A$ be a quantum polynomial ring and let $g$ be a graded algebra automorphism of $A$ of order $p^{m}$ for some prime $p$ and some natural number $m$. Then $A^{g}$ has finite global dimension if and only if $g$ is a quasi-reflection.

We conjecture that a full version of the Shephard-Todd-Chevalley theorem for noncommutative Artin-Schelter regular algebras holds. Some further study about reflection groups and a noncommutative version of the Shephard-Todd-Chevalley theorem will be reported in KKZ1.

\section{General preparations}

In this section we review some background and collect some definitions that we will use in later sections. 
Throughout let $k$ be a commutative base field of characteristic zero. We assume that $k$ is algebraically closed for the convenience of our computation, but this assumption is not necessary for most of the results. All vector spaces, algebras and rings are over $k$. The opposite ring of an algebra $A$ is denoted by $A^{o p}$.

Let $V$ be a finite dimensional vector space over $k$ and let $g$ be a linear transformation of $V$. We call $g$ a reflection of $V$ if $\operatorname{dim} V^{g} \geq \operatorname{dim} V-1$, where $V^{g}$ is the $g$-invariant subspace of $V$. Such a $g$ is also called a pseudo-reflection by many authors [Be, p. 24]. We have dropped the prefix "pseudo" because we will introduce several different kinds of reflections in this paper. Let $k[V]$ denote the symmetric algebra on $V$ - the polynomial ring in $n$ commuting variables where $n=\operatorname{dim} V$. The famous Shephard-Todd-Chevalley theorem gives necessary and sufficient conditions for the fixed ring of a polynomial ring to be a polynomial ring (see $\mathrm{Be}$, Theorem 7.2.1]).

Theorem 1.1 (Shephard-Todd-Chevalley theorem). Suppose $G$ is a finite group acting faithfully on a finite dimensional vector space $V$. Then the fixed subring $k[V]^{G}$ is isomorphic to $k[V]$ if and only if $G$ is generated by reflections of $V$.

A finite group $G \subset G L(V)$ is called a reflection group of $V$ if $G$ is generated by reflections. When the base field is $\mathbb{R}$, a reflection group is also called a Coxeter group. Classifications of reflection groups over different fields are given in Co, ShT, CE.

There are noncommutative algebras that are not rigid, i.e. have fixed subrings isomorphic to themselves. In fact, one can construct an algebra $A$ and a group $G$ of automorphisms of $A$ so that $A^{G}$ is isomorphic to $A$ using any ring $R$ and any graded automorphism $\sigma$ of $R$ with finite order using a skew polynomial extension in the following way.

Example 1.2. If $R$ is an algebra with an automorphism $\sigma$ of order $n$, so that $\sigma^{n+1}=\sigma$, then if we let $\xi$ be an $(n+1)$-st root of unity and extend $\sigma$ to the skew polynomial extension $A=R[z ; \sigma]$ by $\left.g\right|_{R}=I d_{R}$ and $g(z)=\xi z$, then the fixed subring $A^{G}=R\left[z^{n+1} ; \sigma^{n+1}\right] \cong A$ for $G=\langle g\rangle$. We note that if $R$ is Artin-Schelter regular (defined below), then so is $A$.

On the other hand, Alev-Polo's result [Theorem 0.1 and results in [Sm1, AP, Jos, suggest that it is rare that a noncommutative ring $A$ is isomorphic to a fixed subring $A^{G}$ for a finite group $G$. The motivation for this paper is the following question.

Question 1.3. Under what conditions on the algebra $A$ and the group $G$ is $A$ isomorphic to $A^{G}$ ?

Our focus is on graded algebras and graded automorphisms since some combinatorial structures of graded rings and their fixed subrings can be used to study this problem. It follows from the Shephard-Todd-Chevalley theorem that the commutative graded polynomial ring $k[V]$ can be isomorphic to its fixed subrings. Hence it is expected that some version of the Shephard-Todd-Chevalley theorem will hold for "somewhat commutative" polynomial rings. We will present some examples that illustrate this idea [Examples 4.4, 5.4 and 6.6.

In the rest of this section we review some properties of the Hilbert series of an algebra, the trace of an automorphism, and Artin-Schelter regular algebras, as well as some techniques from invariant theory that will be used in this paper. 
Throughout let $A$ be a connected graded algebra, namely,

$$
A=k \oplus A_{1} \oplus A_{2} \oplus \cdots,
$$

where each $A_{i}$ is finite dimensional and $A_{i} A_{j} \subseteq A_{i+j}$ for all $i, j$. The Hilbert series of $A$ is defined to be the formal power series

$$
H_{A}(t)=\sum_{i \geq 0} \operatorname{dim} A_{i} t^{i} \in \mathbb{Z}[[t]] .
$$

The Hilbert series of a graded $A$-module is defined similarly. Let $\operatorname{Autgr}_{\mathrm{gr}}(A)$ be the group of graded algebra automorphisms of $A$. For every $g \in \operatorname{Aut}_{\text {gr }}(A)$, the trace of $g$ [JiZ] is defined to be

$$
\operatorname{Tr}_{A}(g, t)=\sum_{i \geq 0} \operatorname{tr}\left(\left.g\right|_{A_{i}}\right) t^{i} \in k[[t]] .
$$

It is obvious that $\operatorname{Tr}_{A}\left(I d_{A}, t\right)=H_{A}(t)$, and the converse is clearly true for $g$ of finite order when char $k=0$. In the next section we will define our generalization of the notion of a "reflection" in terms of the trace of the automorphism.

If $g$ has finite order, then $\operatorname{Tr}_{A}(g, t)$ is in $\mathbb{Q}\left(\zeta_{n}\right)[[t]]$, where $\mathbb{Q}\left(\zeta_{n}\right)$ is the cyclotomic field generated by the primitive $n$-th roots of unity, $\zeta_{n}=e^{2 \pi i / n}$. For each integer $p$ such that $(p, n)=1$, there is an automorphism of $\mathbb{Q}\left(\zeta_{n}\right) / \mathbb{Q}$ determined by

$$
\Xi_{p}: \zeta_{n} \rightarrow \zeta_{n}^{p}
$$

and the Galois group $G\left(\mathbb{Q}\left(\zeta_{n}\right) / \mathbb{Q}\right)$ is generated by the $\Xi_{p}$. One can easily extend $\Xi_{p}$ to an algebra automorphism of $\mathbb{Q}\left(\zeta_{n}\right)[[t]] / \mathbb{Q}[[t]]$ by applying $\Xi_{p}$ to the coefficients.

Lemma 1.4. Let $g$ be a graded automorphism of $A$ of order $n$. Then, for every $p$ coprime to $n, \operatorname{Tr}_{A}\left(g^{p}, t\right)=\Xi_{p}\left(\operatorname{Tr}_{A}(g, t)\right)$. In particular, if $g \in \operatorname{Aut}_{\mathrm{gr}}(A)$ is of finite order, then $\operatorname{Tr}_{A}\left(g^{-1}, t\right)=\overline{\operatorname{Tr}_{A}(g, t)}$, where for computational purposes we assume $k \subset \mathbb{C}$ and $\bar{f}$ is the series whose coefficients are complex conjugates of the coefficients of $f$.

Proof. We only need to show that $\operatorname{tr}\left(\left.g^{p}\right|_{A_{i}}\right)=\Xi_{p}\left(\operatorname{tr}\left(\left.g\right|_{A_{i}}\right)\right)$ for all $i$. Since $g$ has order $n$, it is diagonalizable. Let $\left\{b_{1}, \cdots, b_{q}\right\}$ be a basis of $A_{i}$ such that

$$
g\left(b_{t}\right)=\zeta_{n}^{w_{t}} b_{t}
$$

for some integer $w_{t}$, for all $t=1, \cdots, q$. For every $p$ coprime to $n, \Xi_{p}$ is an automorphism and

$$
g^{p}\left(b_{t}\right)=\zeta_{n}^{p w_{t}} b_{t}=\Xi_{p}\left(\zeta_{n}^{w_{t}}\right) b_{t} .
$$

Hence $\operatorname{tr}\left(\left.g^{p}\right|_{A_{i}}\right)=\Xi_{p}\left(\operatorname{tr}\left(\left.g\right|_{A_{i}}\right)\right)$. The second part follows since for a root of unity $\zeta^{-1}=\bar{\zeta}$.

We will use this lemma when $\operatorname{Tr}_{A}(g, t)$ is a rational function, viewed as an infinite power series.

The Gelfand-Kirillov dimension of an algebra $A$ is denoted by GKdim $A$; it is related to the rate of growth of the graded pieces $A_{n}$ of $A$ (see [KL]). The commutative polynomial ring $k\left[x_{1}, \cdots, x_{n}\right]$ has GKdim $=n$. The Gelfand-Kirillov dimension of an $A$-module is defined similarly. Let $\underline{\operatorname{Ext}}_{A}(M, N)$ be the usual Ext-group of graded $A$-modules $M$ and $N$ with $\mathbb{Z}$-grading as defined in [AZ, p. 240]. 
Definition 1.5. A connected graded algebra A is called Artin-Schelter Gorenstein if the following conditions hold:

(a) $A$ has graded injective dimension $d<\infty$ on the left and on the right,

(b) $\operatorname{Ext}_{A}^{i}(k, A)=\operatorname{Ext}_{A^{o p}}^{i}(k, A)=0$ for all $i \neq d$, and

(c) $\underline{\operatorname{Ext}}_{A}^{d}(k, A) \cong \underline{\operatorname{Ext}}_{A^{o p}}^{d}(k, A) \cong k(l)$ for some $l$.

If in addition,

(d) $A$ has finite (graded) global dimension, and

(e) $A$ has finite Gelfand-Kirillov dimension,

then $A$ is called Artin-Schelter regular (or regular for short) of dimension $d$.

Note that the polynomial rings $k\left[x_{1}, x_{2}, \cdots, x_{n}\right]$ for $n \geq 0$, with $\operatorname{deg} x_{i}>0$, are Artin-Schelter regular of dimension $n$, and these are the only commutative Artin-Schelter regular algebras, so Artin-Schelter regular algebras are natural generalizations of commutative polynomial rings.

For (Artin-Schelter) regular algebras we can say more about the trace of an automorphism.

Lemma 1.6. Let $A$ be regular and let $g \in \operatorname{Aut}_{\mathrm{gr}}(A)$.

(a) [JiZ, Theorem 2.3(4)] $\operatorname{Tr}_{A}(g, t)$ is equal to $1 / e_{g}(t)$, where $e_{g}(t)$ is a polynomial in $k[t]$ with $e_{g}(0)=1$. We call $e_{g}(t)$ the Euler polynomial of $g$.

(b) [StZ, Proposition 3.1(3)] $H_{A}(t)=1 / e(t)$ where $e(t)$ is an integral polynomial. The polynomial $e(t)$ is called the Euler polynomial of A. Furthermore $e(t)$ is a product of cyclotomic polynomials.

(c) [StZ, Corollary 2.2] The multiplicity of $t=1$ as a root of the Euler polynomial of $A$ is the GKdim $A$.

(d) [JiZ, Theorem 3.1] The polynomials $e(t)$ and $e_{g}(t)$ have the same degree.

(e) Suppose $g$ has finite order and $\operatorname{Tr}_{A}(g, t)=e_{g}(t)^{-1}$. Then the zeroes of the polynomial $e_{g}(t)$ are all roots of unity.

Proof. Only the second assertion in (b) and assertion (e) are new.

(b) By [StZ, Corollary 2.2], all the zeroes of the polynomial $e(t)$ appearing in Lemma 1.6(b) are roots of unity. Since $e(t) \in \mathbb{Z}[t]$, therefore $e(t)$ is a product of cyclotomic polynomials.

(e) Let $n$ be the order of $g$. Let $p$ be any integer $0<p<n$ coprime to $n$. By Lemma 1.4. $\operatorname{Tr}_{A}\left(g^{p}, t\right)=\Xi_{p}\left(\operatorname{Tr}_{A}(g, t)\right)$. Let $e_{p}(t)=\left(\operatorname{Tr}_{A}\left(g^{p}, t\right)\right)^{-1}$ for all $p$. By [JiZ, Proposition 3.3], every zero of $e_{p}(t)$ has absolute value 1. Now let

$$
f(t)=\prod_{(p, n)=1} e_{p}(t)=\prod_{(p, n)=1} \Xi_{p}\left(\operatorname{Tr}_{A}(g, t)\right)^{-1},
$$

where the notation $(p, n)=1$ means the set of integers $p$ such that $0<p<n$ and that $p$ is coprime to $n$. Since all coefficients of $\Xi_{p}\left(\operatorname{Tr}_{A}(g, t)\right)^{-1}$ are in $\mathbb{Z}\left[\zeta_{n}\right]$, $f(t) \in \mathbb{Z}\left(\zeta_{n}\right)[t]$. By the definition of $f(t), \Xi_{p}(f(t))=f(t)$. Since the coefficients of $f(t)$ are fixed by all elements of the Galois group $G\left(\mathbb{Q}\left(\zeta_{n}\right) / \mathbb{Q}\right)$ therefore $f(t) \in \mathbb{Q}[t] \cap \mathbb{Z}\left[\zeta_{n}\right][t]=\mathbb{Z}[t]$. Since every zero of $f(t)$ is an algebraic integer with it and all its conjugates having absolute value 1 , it follows from Mo, Corollary 2.38, p. 90] that every zero of $e(t)$ (and hence of $e_{p}(t)$ ) is a root of unity.

Next we consider the multiplicity of $t=1$ as a root of the Euler polynomial of a finite graded automorphism $g$ of a regular domain $A$. We show that this 
multiplicity is bounded by the GKdim $A$, and can be equal to GKdim $A$ only when $g$ is the identity automorphism.

Lemma 1.7. Let $A$ be a connected graded finitely generated algebra, and let $M$ be a graded finitely generated right $A$-module of GKdim $M=n$. Let $g$ be a graded vector space automorphism of $M$ that has finite order and $\operatorname{Tr}_{M}(g, t)=p(t) / q(t)$, where the roots of $q(t)$ are roots of unity. Then the multiplicity of 1 as a root of $q(t)$ is $\leq n$.

Proof. Assume to the contrary that the multiplicity of 1 as a root of $q(t)$ is $\geq n+1$. Let $H_{M}(t)=\sum h_{i} t^{i}$ be the Hilbert series of $M$, and let $\operatorname{Tr}_{M}(g, t)=\sum m_{i} t^{i}$ be the trace function of $g$ on $M$. We note that $\left|m_{i}\right| \leq h_{i}$ for all $i$ since $g$ has finite order so that the eigenvalues of $g$ are roots of unity. As in the proof of [ATV2, Proposition 2.21 ], let $p$ be the highest order of any pole of $\operatorname{Tr}_{M}(g, t)$, and express all roots of $q(t)$ as powers of a primitive $N$ th root of unity $\zeta$. We have

$$
\operatorname{Tr}_{M}(g, t)=\sum_{s, j} \frac{c_{s, j}}{\left(1-\zeta^{s} t\right)^{j}}+f(t)
$$

where $s=0, \ldots, N, j=1, \ldots, p$, and for sufficiently large $i$ we have

$$
\begin{aligned}
m_{i} & =\sum_{s, j} c_{s, j}\left(\begin{array}{c}
i+j-1 \\
j-1
\end{array}\right) \zeta^{s i} \\
& =\left(\sum_{s} c_{s, p} \zeta^{s i}\right) \frac{i^{p-1}}{(p-1) !}+\text { terms of lower degree in } i,
\end{aligned}
$$

and the coefficients cycle through $N$ polynomials. Not all the $c_{s, p}$ are zero, so the leading coefficients $\sum c_{s, p} \zeta^{s i}$ are not all zero. Hence there is a subsequence $m_{i_{0}+N i}$ with $\left|m_{i_{0}+N i}\right| \geq K(N i)^{p-1}$ for all $i \geq 1$, for some constant $K$. Since $h_{i} \geq\left|m_{i}\right|$ for all $i$,

$$
\sum_{j \leq i_{0}+N i} h_{i} \geq \sum_{s \leq i}\left|m_{i_{0}+N s}\right| \geq K N^{p-1} \sum_{s \leq i} s^{p-1} \geq K^{\prime} i^{p} \geq K^{\prime \prime}\left(i_{0}+N i\right)^{p}
$$

for some $K^{\prime}, K^{\prime \prime}>0$. Since $A$ is a finitely generated algebra and $M$ is a finitely generated $A$-module, GKdim $M \geq p$. This contradicts the fact that GKdim $M=n$ and $p \geq n+1$.

Proposition 1.8. Let $A$ be a regular domain. If $g \in \operatorname{Autgr}_{\mathrm{gr}}(A)$ has finite order, and if its Euler polynomial has $t=1$ as a root of multiplicity equal to the GKdim $A$, then $g$ is the identity.

Proof. Suppose that the Euler polynomial of $g$ has $t=1$ a root of multiplicity equal to $\operatorname{GKdim} A$, but that $g$ is not the identity. Then $g$ has an eigenvalue $\lambda \neq 1$ and an element $x \in A$ with $g(x)=\lambda x$. Let $M=A / x A$ and let $\bar{g}$ be the induced graded vector space automorphism of $M$. Then

$$
\operatorname{Tr}_{M}(\bar{g}, t)=(1-\lambda t) \operatorname{Tr}_{A}(g, t),
$$

and the order of the pole of $\operatorname{Tr}_{M}(\bar{g}, t)$ at $t=1$ is equal to the order of the pole of $\operatorname{Tr}_{A}(g, t)$ at $t=1$, which is by assumption the GKdim $A$. But GKdim $A>$ GKdim $M$ by [MR, Proposition 8.3.5], contradicting Lemma 1.7 
Associated to a graded automorphism $g$ of an Artin-Schelter Gorenstein algebra $A$ is a constant $\operatorname{hdet}_{A} g$ defined by Jørgensen and Zhang [JoZ, and the map $\operatorname{hdet}_{A}$ : $\operatorname{Aut}_{\mathrm{gr}}(A) \rightarrow k^{\times}$defines a group homomorphism. It follows from [JoZ, Lemma 2.6 and Theorem 4.2] that when $A$ is a regular algebra, then the hdet $g$ can be computed from the trace of $g$ : since $\operatorname{Tr}_{A}(g, t)$ is a rational function in $t$ it can be written as a Laurent series in $t^{-1}$, and we can write

$$
\operatorname{Tr}_{A}(g, t)=(-1)^{d}(\text { hdet } g)^{-1} t^{-l}+\text { lower degree terms, }
$$

where $d$ and $l$ are as in Definition 1.5(d). By [JoZ, Theorem 3.3], if $G$ is a finite group of graded automorphisms acting on an Artin-Schelter Gorenstein ring $A$, and if the homological determinant of $g$ satisfies hdet $g=1$ for all $g \in G$, then the fixed subring $A^{G}$ is Artin-Schelter Gorenstein.

Let $e(t)=a_{n} t^{n}+a_{n-1} t^{n-1}+\cdots+a_{1} t+a_{0}$ be an integral polynomial with $a_{0}=1$. We say $e(t)$ is a palindrome polynomial if $a_{n-i}=a_{i}$ for all $i$ and a skew palindrome polynomial if $a_{n-i}=-a_{i}$ for all $i$. If $e(t)$ is a skew palindrome polynomial, then $e(1)=0$. Any polynomial which is a product of cyclotomic polynomials is either a palindrome or a skew palindrome polynomial.

Lemma 1.9. Let $e(t)$ be a palindrome polynomial of degree $n$. Then $e^{\prime}(1)=$ $n e(1) / 2$, where $e^{\prime}(t)$ is the derivative of $e(t)$.

Proof. First we suppose $n$ is odd and let $m=(n-1) / 2$. Since $a_{n-i}=a_{i}$ for all $i$,

$$
e(t)=\sum_{i=0}^{n} a_{i} t^{i}=\sum_{i=0}^{m} a_{i}\left(t^{i}+t^{n-i}\right) .
$$

Then

$$
e(1)=\sum_{i=0}^{m} a_{i}(1+1)=2 \sum_{i=0}^{m} a_{i}
$$

and

$$
e^{\prime}(1)=\sum_{i=0}^{m} a_{i}\left(i 1^{i-1}+(n-i) 1^{n-i-1}\right)=n \sum_{i=0}^{m} a_{i}=\frac{n}{2} 2 \sum_{i=0}^{m} a_{i}=\frac{n}{2} e(1) .
$$

If $n$ is even, let $f(t)=e(t)(1+t)$. Then $f(t)$ is a palindrome polynomial of even degree. By the above proof, the assertion holds for $f(t)$. Using $f(t)=e(t)(1+t)$ we see that

$$
e^{\prime}(1) 2+e(1)=f^{\prime}(1)=\frac{n+1}{2} f(1)=(n+1) e(1),
$$

which implies that $e^{\prime}(1)=n e(1) / 2$.

The following two lemmas are well known. We say a subring $B$ of $A$ is cofinite if $A_{B}$ and ${ }_{B} A$ are finite $B$-modules.

Lemma 1.10. Suppose $A$ is a graded algebra of finite global dimension and $B$ is a graded subring of $A$.

(a) If ${ }_{B} A$ is free, then $B$ has finite global dimension. If ${ }_{B} A$ is finitely generated, then gldim $A=\operatorname{gldim} B$.

(b) If gldim $B=\operatorname{gldim} A$ and ${ }_{B} A$ is finitely generated, then ${ }_{B} A$ is free.

(c) Suppose $B$ is a cofinite subring of $A$ with $\operatorname{gldim} B<\infty$. If $A=B \oplus C$ as a B-bimodule, then $A$ is regular if and only if $B$ is. 
(d) If $A$ is noetherian and regular and $B$ is a factor ring of $A$ with finite global dimension, then $B$ is regular.

(e) If $\operatorname{deg} y=1$, then $A$ is noetherian and regular if and only if $A /(y)$ is.

Here is a list of well known facts about fixed subrings.

Lemma 1.11. Let $A$ be a noetherian connected graded algebra and let $G$ be a finite subgroup of $\operatorname{Autgr}_{\mathrm{gr}}(A)$.

(a) [Mon1, Corollaries 1.12 and 5.9] $A^{G}$ is noetherian and $A$ is finite over $A^{G}$ on the left and on the right. As a consequence, GKdim $A=\operatorname{GKdim} A^{G}$.

(b) [Mon1, Corollary 1.12] $A=A^{G} \oplus C$ as an $A^{G}$-bimodule.

(c) (Molien's theorem) [JiZ, Lemma 5.2]

$$
H_{A^{G}}(t)=\frac{1}{|G|} \sum_{g \in G} \operatorname{Tr}_{A}(g, t) .
$$

(d) Suppose $A$ and $A^{G}$ are both regular. Then $\operatorname{gldim} A=\operatorname{gldim} A^{G}$ and $A$ is free over $A^{G}$ on both sides.

Proof. (d) For any noetherian regular algebra $A$ of global dimension $d$, we have $\operatorname{cd}(A)=d-1[\mathrm{AZ}$, Theorem 8.1(4)], where

$$
\operatorname{cd}(A)=\operatorname{cd}(\operatorname{Proj} A)=\max \left\{i \mid \underline{\mathrm{H}}_{\operatorname{Proj} A}^{i}(A) \neq 0\right\}
$$

(see [AZ, p. 272 and p. 276] for the definitions). By [AZ, Corollary 8.4(1)], $\operatorname{cd}(A) \leq$ $\operatorname{cd}\left(A^{G}\right)$. Since $A=A^{G} \oplus C$ as an $A^{G}$-bimodule (see part (b)),

$$
\begin{aligned}
\operatorname{cd}(A) & =\max \left\{i \mid \underline{\mathrm{H}}_{\operatorname{Proj} A}^{i}(A) \neq 0\right\} \\
& =\max \left\{i \mid \underline{\mathrm{H}}_{\operatorname{Proj} A}^{i}\left(A^{G} \oplus C\right) \neq 0\right\} \\
& =\max \left\{i \mid \underline{\mathrm{H}}_{\operatorname{Proj} A^{G}}^{i}\left(A^{G} \oplus C\right) \neq 0\right\} \quad[\text { AZ, Theorem 8.3(3)] } \\
& \geq \max \left\{i \mid \underline{\mathrm{H}}_{\operatorname{Proj} A^{G}}\left(A^{G}\right) \neq 0\right\} \\
& =\operatorname{cd}\left(A^{G}\right) .
\end{aligned}
$$

Therefore $\operatorname{cd}(A)=\operatorname{cd}\left(A^{G}\right)$ and

$$
\operatorname{gldim} A=\operatorname{cd}(A)+1=\operatorname{cd}\left(A^{G}\right)+1=\operatorname{gldim} A^{G} .
$$

The rest follows from Lemma 1.10(b).

Definition 1.12. Let $A$ be a connected graded algebra. If $A$ is a noetherian, regular graded domain of global dimension $n$ and $H_{A}(t)=(1-t)^{-n}$, then we call $A$ a quantum polynomial ring of dimension $n$.

By [Sm2, Theorem 5.11], a quantum polynomial ring is Koszul and hence it is generated in degree 1. The GK-dimension of a quantum polynomial ring of global dimension $n$ is $n$. In general, if $A$ is finitely generated and $H_{A}(t)=\left((1-t)^{n} p(t)\right)^{-1}$ for some polynomial $p(t)$ with $p(1) \neq 0$, then the GK-dimension of $A$ is equal to $n$. A quantum polynomial ring of dimension 2 is isomorphic to either:

(i) $k_{q}[x, y]:=k\langle x, y\rangle /(x y-q y x)$ for some $0 \neq q \in k$, or

(ii) $k_{J}[x, y]:=k\langle x, y\rangle /\left(x y-y x-x^{2}\right)$.

Quantum polynomial rings of dimension 3 were classified in AS, ATV1]. There are many examples of quantum polynomial rings of higher dimensions, but their classification has not yet been completed. 


\section{QUASI-REFLECTIONS}

The Shephard-Todd-Chevalley theorem suggests that if a fixed subring $A^{G}$ of a regular algebra $A$ is still regular, then $G$ is some kind of a reflection group. In the commutative case the reflection is defined on the generating space of $k[V]$. In the noncommutative case, this becomes a complicated issue as many examples indicate. The following easy fact (see [JiZ, (1-1)]) suggests one possible definition of reflection.

Lemma 2.1. Let $V$ be a vector space of dimension $n$. Let $g$ be a linear transformation of $V$ of finite order, extending to an algebra automorphism of $A:=k[V]$. Then $g$ is a reflection of $V$ if and only if there is $\xi \in k$ with

$$
\operatorname{Tr}_{A}(g, t)=\frac{1}{(1-t)^{n-1}(1-\xi t)}
$$

By Lemma 1.7 and Proposition [1.8, we have seen that if $g \neq 1$ is a finite order graded automorphism of a regular algebra $A$, then the order of the pole at $t=1$ in $\operatorname{Tr}_{A}(g, t)$ must be strictly less than the order of the pole at $t=1$ in $H_{A}(t)$, which is the GKdim $A$. We will call those graded automorphisms whose trace has a pole at $t=1$ of order GKdim $A-1$ quasi-reflections.

Definition 2.2. Let $A$ be a regular graded algebra such that

$$
H_{A}(t)=\frac{1}{(1-t)^{n} p(t)}
$$

where $p(1) \neq 0$. Let $g$ be a graded algebra automorphism of $A$. We say that $g$ is a quasi-reflection of $A$ if

$$
\operatorname{Tr}_{A}(g, t)=\frac{1}{(1-t)^{n-1} q(t)}
$$

for $q(1) \neq 0$. If $A$ is a quantum polynomial ring, then $H_{A}(t)=(1-t)^{-n}$. In this case $g$ is a quasi-reflection if and only if

$$
\operatorname{Tr}_{A}(g, t)=\frac{1}{(1-t)^{n-1}(1-\xi t)}
$$

for some $\xi \neq 1$. (Note that we have chosen not to call the identity map a quasireflection.)

The next example shows that if we use the definition of a "reflection" from the commutative case, then the condition that $G$ is generated by "reflections" is neither necessary nor sufficient for the fixed subring of a noncommutative quantum polynomial ring to be regular.

Example 2.3. Let $A$ be the regular algebra $k\langle x, y\rangle /\left(x^{2}-y^{2}\right)$. This is a quantum polynomial ring and is isomorphic to $k_{-1}\left[b_{1}, b_{2}\right]$.

(a) Let $h$ be the automorphism of $A$ determined by

$$
h(x)=-x \quad \text { and } \quad h(y)=y .
$$

Hence $h$ is a reflection of the generating space $A_{1}:=k x \oplus k y$. Since $A$ has a $k$-linear basis

$$
\left\{(y x)^{i} y^{j} \mid i, j \geq 0\right\} \cup\left\{x(y x)^{i} y^{j} \mid i, j \geq 0\right\}
$$


we can compute the trace easily:

$$
\operatorname{Tr}_{A}(h, t)=\frac{1}{1+t^{2}}, \quad \operatorname{Tr}_{A}(I d, t)=\frac{1}{(1-t)^{2}} .
$$

By definition, $h$ is not a quasi-reflection. Furthermore, the fixed subring $A^{h}$ is not regular because its Hilbert series is

$$
H_{A^{h}}(t)=\frac{1}{2}\left(\operatorname{Tr}_{A}(h, t)+\operatorname{Tr}_{A}(I d, T)\right)=\frac{1-t+t^{2}}{(1-t)^{2}\left(1+t^{2}\right)} \neq \frac{1}{p(t)} .
$$

However, by [JoZ, Theorem 6.4 or Theorem 3.3], $A^{h}$ is Artin-Schelter Gorenstein.

To summarize, there is an automorphism $h$ of $A$ with order 2 such that $\left.h\right|_{A_{1}}$ is a reflection, but $h$ is not a quasi-reflection and the fixed subring $A^{h}$ has infinite global dimension. Consequently, $A^{h} \approx A$. If we believe that a reflection of $A$ should give rise to a regular fixed subring as in the Shephard-Todd-Chevalley theorem, then we should not think of $h$ as a reflection of $A$.

(b) Let $g$ be the automorphism of $A$ determined by

$$
g(x)=i x \text { and } g(y)=-i y .
$$

Hence $\left.g\right|_{A_{1}}$ is not a reflection (and neither is $\left.g^{2}\right|_{A_{1}}$ ). Using the $k$-linear basis in (2.3.1), we can compute the trace easily:

$$
\operatorname{Tr}_{A}(g, t)=\operatorname{Tr}\left(g^{3}, t\right)=\frac{1}{1-t^{2}}, \quad \operatorname{Tr}_{A}\left(g^{2}, t\right)=\frac{1}{(1+t)^{2}}, \quad \operatorname{Tr}_{A}(I d, t)=\frac{1}{(1-t)^{2}} .
$$

So $g$ is a quasi-reflection, but $g^{2}$ is not.

Using the $k$-linear basis above again, one can check that $A^{g}=k[x y, y x] \cong k[t, s]$. Hence $A^{g}$ is regular (although not isomorphic to $A$ ). But $A^{g^{2}}$ is not regular by a Hilbert series computation.

To summarize, there is a quasi-reflection $g$ such that $\left.g\right|_{A_{1}}$ is not a reflection. Since the fixed subring $A^{g}$ is regular, we should think of $g$ as a reflection. On the other hand, the automorphism $g^{2}$ is not a quasi-reflection and $A^{g^{2}}$ is not regular. So we should not think of $g^{2}$ as a reflection. This phenomenon is quite different from the commutative case (where the square of a reflection is a reflection), and it conflicts with our intuition.

Next we prove some general results relating quasi-reflections to the regularity of the fixed rings. The theorem below justifies our definition of quasi-reflection.

Theorem 2.4. Let $A$ be noetherian and regular. Let $G$ be a finite subgroup of $\operatorname{Aut}_{\mathrm{gr}}(A)$. If $A^{G}$ has finite global dimension, then $G$ contains a quasi-reflection.

Proof. We show that the assumption that $G$ does not contain a quasi-reflection leads to a contradiction.

Since $A$ is regular, the Hilbert series of $A$ is

$$
H_{A}(t)=\frac{1}{(1-t)^{n} p(t)}
$$

with $p(1) \neq 0$, where $n=\operatorname{GKdim} A$.

By Lemma 1.11(a), $A^{G}$ is noetherian and $A$ is finite over $A^{G}$ on the left and on the right, and $\mathrm{GKdim} A=\mathrm{GKdim} A^{G}$. Since $A^{G}$ has finite global dimension, the Hilbert series of $A^{G}$ is of the form

$$
H_{A^{G}}(t)=\frac{1}{e(t)}=\frac{1}{(1-t)^{n} q(t)}
$$


By Lemma 1.11(d), $A$ is free finite over $A^{G}$. Hence $H_{A}(t)=f(t) H_{A^{G}}(t)$ for some polynomial $f(t)$ with nonnegative integer coefficients. Clearly $q(t)=p(t) f(t)$. Let $m=\operatorname{deg} p(t)$ and $n=\operatorname{deg} q(t)$. Then $n-m=\operatorname{deg} f(t)>0$.

Expanding $H_{A}(t)$ into a Laurent series about $t=1$ we have

$$
H_{A}(t)=\frac{1}{(1-t)^{n}} \frac{1}{p(1)}+\frac{1}{(1-t)^{n-1}} \frac{p^{\prime}(1)}{p^{2}(1)}+\text { higher degree terms. }
$$

Similarly,

$$
H_{A^{G}}(t)=\frac{1}{(1-t)^{n}} \frac{1}{q(1)}+\frac{1}{(1-t)^{n-1}} \frac{q^{\prime}(1)}{q^{2}(1)}+\text { higher degree terms. }
$$

From Molien's theorem [Lemma 1.11(c)], we have that

$$
H_{A^{G}}(t)=\frac{1}{|G|} \sum_{g \in G} \operatorname{Tr}_{A}(g, t) \text {. }
$$

If we expand this expression into a Laurent series around $t=1$, since $G$ does not contain any quasi-reflections, by Lemma 1.7 and Proposition 1.8 the Laurent series of $\operatorname{Tr}(g, t)$ has lowest possible degree term $(1-t)^{-(n-2)}$. Hence the first terms of the sum come entirely from the trace of the identity $\operatorname{Tr}_{A}\left(I d_{A}, t\right)=H_{A}(t)$. Hence

$$
H_{A^{G}}(t)=\frac{1}{|G|}\left[\frac{1}{(1-t)^{n}} \frac{1}{p(1)}+\frac{1}{(1-t)^{n-1}} \frac{p^{\prime}(1)}{p^{2}(1)}+\text { higher degree terms }\right] .
$$

Equating coefficients in the two expressions for $H_{A^{G}}(t)$ we have that

$$
q(1)=|G| p(1) \quad \text { and } \quad \frac{q^{\prime}(1)}{q(1)^{2}}=\frac{1}{|G|} \frac{p^{\prime}(1)}{p(1)^{2}} .
$$

Since $p(t)$ and $q(t)$ are products of cyclotomic polynomials, they are palindrome polynomials. By Lemma [1.9,

$$
2 p^{\prime}(1)=m p(1) \quad \text { and } \quad 2 q^{\prime}(1)=n q(1) .
$$

Hence we have

$$
\frac{q^{\prime}(1)}{(q(1))^{2}}=\frac{n}{2 q(1)} \quad \text { and } \quad \frac{p^{\prime}(1)}{|G|(p(1))^{2}}=\frac{m}{2|G|(p(1))}=\frac{m}{2 q(1)},
$$

and so

$$
\frac{n}{2 q(1)}=\frac{m}{2 q(1)}
$$

gives $n=m$, a contradiction.

The number of quasi-reflections in $G$ can also be computed.

Theorem 2.5. Suppose $A$ is a quantum polynomial ring, and let $G$ be a finite subgroup of $\operatorname{Autgr}_{\mathrm{gr}}(A)$. Denote the number of quasi-reflections in $G$ by $r$.

(a) If $H_{A^{G}}(t)$ is expanded into a Laurent series around $t=1$, then the coefficient of $(1-t)^{-(n-1)}$ is given by $r /(2|G|)$.

(b) Suppose $A^{G}$ is regular and $H_{A^{G}}(t)=\left((1-t)^{n} q(t)\right)^{-1}$. Then $q(1)=|G|$ and $r=\operatorname{deg} q(t)$. 
Proof. (a) Let $g_{1}, \cdots, g_{r}$ be the quasi-reflections (that are not the identity) in $G$, and let $h_{1}, \cdots, h_{s}$ be the nonidentity elements of $G$ that are not quasi-reflections. By Lemma 1.6. for all $g \in G, \operatorname{Tr}_{A}(g, t)=1 / e_{g}(t)$ where $e_{g}(t)$ has degree $n$. Suppose now $g$ is a quasi-reflection. Then

$$
\operatorname{Tr}_{A}(g, t)=\frac{1}{(1-t)^{n-1}(1-\lambda t)},
$$

where $\lambda \neq 1 \in k$. By Lemma 1.6(d), $\lambda$ is a root of unity. Thus the Laurent expansion of $\operatorname{Tr}_{A}(g, t)$ around $t=1$ is given by

$$
\operatorname{Tr}_{A}(g, t)=\frac{1}{(1-t)^{n-1}}\left[\frac{1}{1-\lambda}+(1-t) a_{1}+\cdots\right] .
$$

By Lemma 1.4, the Laurent expansion of $\operatorname{Tr}_{A}\left(g^{-1}, t\right)$ is given by

$$
\operatorname{Tr}_{A}\left(g^{-1}, t\right)=\overline{\operatorname{Tr}_{A}(g, t)}=\frac{1}{(1-t)^{n-1}}\left[\frac{1}{1-\bar{\lambda}}+(1-t) \overline{a_{1}}+\cdots\right] .
$$

In particular, $g^{-1}$ is again a quasi-reflection. This also shows that if $g$ has order 2, then

$$
\operatorname{Tr}_{A}(g, t)=\frac{1}{(1-t)^{n-1}(1+t)}=\frac{1}{(1-t)^{n-1}}\left[\frac{1}{2}+(1-t) a_{1}+\cdots\right] .
$$

Note that

$$
\frac{1}{1-\lambda}+\frac{1}{1-\bar{\lambda}}=\frac{1-\bar{\lambda}+1-\lambda}{(1-\lambda)(1-\bar{\lambda})}=\frac{1-\bar{\lambda}+1-\lambda}{1-\lambda-\bar{\lambda}+\lambda \bar{\lambda}}=1
$$

since $\lambda \bar{\lambda}=1$. Now let $h$ be a nonidentity element in $G$ that is not a quasi-reflection. Then the Laurent expansion of its trace is of the form

$$
\operatorname{Tr}_{A}(h, t)=\frac{1}{(1-t)^{n-2}}\left(c_{0}+c_{1}(1-t)+\cdots\right) .
$$

By Molien's theorem [Lemma 1.11(c)] we have

$$
H_{A^{G}}(t)=\frac{1}{|G|} \sum_{g \in G} \operatorname{Tr}_{A}(g, t)=\frac{1}{|G|}\left[\frac{1}{(1-t)^{n}}+\sum_{i=1}^{r} \operatorname{Tr}_{A}\left(g_{i}, t\right)+\sum_{j=1}^{s} \operatorname{Tr}_{A}\left(h_{j}, t\right)\right] .
$$

We see that the only contributions to the coefficient of $\frac{1}{(1-t)^{n-1}}$ come from the $\sum_{i=1}^{r} \operatorname{Tr}_{A}\left(g_{i}, t\right)$ term. By grouping each $g_{i}$ with its inverse, we see that the coefficient is exactly $r /(2|G|)$. (b) Expanding $H_{A^{G}}(t)$ around $t=1$, we have

$$
H_{A^{G}}(t)=\frac{1}{(1-t)^{n}} \frac{1}{q(1)}+\frac{1}{(1-t)^{n-1}} \frac{q^{\prime}(1)}{q^{2}(1)}+\cdots .
$$

Comparing the coefficients of $1 /(1-t)^{n}$ and $1 /(1-t)^{n-1}$ in (2.5.1) and (2.5.2), we see that

$$
\frac{1}{|G|}=\frac{1}{q(1)} \quad \text { and } \quad \frac{r}{2|G|}=\frac{q^{\prime}(1)}{q^{2}(1)} .
$$

Combining with Lemma 1.9, we obtain $r=\operatorname{deg} q(t)$.

The following lemma will be used in the next section. 
Lemma 2.6 ([JiZ, Theorem 2.3.2]). Let $A$ be a noetherian regular algebra and let $g$ be a graded algebra automorphism of $A$. Suppose $B$ is a factor ring of $A$ such that $g$ induces an algebra automorphism $g^{\prime}$ of $B$. Then $\operatorname{Tr}_{B}\left(g^{\prime}, t\right)=p(t) \operatorname{Tr}_{A}(g, t)$, where $p(t)$ is a polynomial of $t$ with $p(0)=1$.

\section{QUASI-REFLECTIONS OF QUANTUM POLYNOMIAL RINGS}

In this section we will classify all possible quasi-reflections of a quantum polynomial ring. The proof of the following main result requires several lemmas.

Theorem 3.1. Let $A$ be a quantum polynomial ring of global dimension $n$. If $g \in \operatorname{Aut}_{\mathrm{gr}}(A)$ is a quasi-reflection of finite order, then $g$ is in one of the following two cases:

(a) There is a basis of $A_{1}$, say $\left\{b_{1}, \cdots, b_{n}\right\}$, such that $g\left(b_{j}\right)=b_{j}$ for all $j \geq 2$ and $g\left(b_{1}\right)=\xi b_{1}$. Namely, $\left.g\right|_{A_{1}}$ is a reflection.

(b) The order of $g$ is 4 and there is a basis of $A_{1}$, say $\left\{b_{1}, \cdots, b_{n}\right\}$, such that $g\left(b_{j}\right)=b_{j}$ for all $j \geq 3$ and $g\left(b_{1}\right)=i b_{1}$ and $g\left(b_{2}\right)=-i b_{2}\left(\right.$ where $\left.i^{2}=-1\right)$.

We start with a lemma about sums of roots of unity.

Lemma 3.2. Every solution of the following system

$$
n=x_{1}+x_{2}+\cdots+x_{n+2}
$$

with each $x_{i}$ being a root of unity, but not 1 , is in one of the following cases:

(a) $n=0, x_{1}=\xi$ and $x_{2}=-\xi$ where $\xi$ is a root of 1 , which is not \pm 1 .

(b) $n=2, x_{1}=x_{2}=\zeta_{6}$ and $x_{3}=x_{4}=\overline{\zeta_{6}}$ and all possible permutations.

Proof. First we claim that $x_{i}$ cannot be -1 . If $x_{i}=-1$, say $x_{n+2}=-1$, the equation becomes

$$
n=x_{1}+\cdots+x_{n+1}-1
$$

or

$$
n+1=x_{1}+\cdots+x_{n+1} .
$$

Since every $x_{i}$ is a root of 1 , but not 1 , the real part of each $x_{i}$ is strictly less than 1 , and there is no solution to the above equation. Thus we proved our claim that $x_{i} \neq-1$ for all $i$.

Let $w_{i}$ be the order of $x_{i}$ and let $w$ be the gcd of the $w_{i}$. Since $x_{i} \neq-1, w_{i} \geq 3$. The Galois group of $\mathbb{Q}\left(\zeta_{w}\right)$ over $\mathbb{Q}$ is $(\mathbb{Z} / w \mathbb{Z})^{*}$. For every $p$ coprime to $w$, let $\Xi_{p}$ denote the automorphism determined by $\Xi_{p}(\zeta)=\zeta_{w}^{p}$. Let $\Xi$ denote the group $\left\{\Xi_{p} \mid(p, w)=1\right\}=(\mathbb{Z} / w \mathbb{Z})^{*}$. The order of $\Xi$ is $\phi(w)$. Recall that

$$
\sum_{(p, w)=1} \zeta_{w}^{p}=\mu(w):= \begin{cases}(-1)^{t} & \text { if } w \text { is a product of } t \text { distinct primes } \\ 0 & \text { if } w \text { is not square-free }\end{cases}
$$

where the notation $(p, w)=1$ means the set of integers $p$ such that $0<p<$ $w$ and that $p$ is coprime to $w$ (see $\left[\mathrm{HW}\right.$ (16.6.4), p. 239]). Let $\Xi\left[x_{i}\right]$ denote $\sum_{(p, w)=1} \Xi_{p}\left(x_{i}\right)$. Since $\Xi\left[x_{i}\right]$ is stable under the $\Xi$-action, it contains $\phi(w) / \phi\left(w_{i}\right)$ copies of each $w_{i}$-th primitive root of unity. Thus we have

$$
\Xi\left[x_{i}\right]=\frac{\phi(w)}{\phi\left(w_{i}\right)} \sum_{\left(p, w_{i}\right)=1} \zeta_{w_{i}}^{p}=\frac{\phi(w)}{\phi\left(w_{i}\right)} \mu\left(w_{i}\right) .
$$


Applying $\Xi$ to equation (3.2.1) we obtain that

$$
n \phi(w)=\sum_{i=1}^{n+2} \frac{\phi(w)}{\phi\left(w_{i}\right)} \mu\left(w_{i}\right) .
$$

Hence

$$
n=\sum_{i=1}^{n+2} \frac{1}{\phi\left(w_{i}\right)} \mu\left(w_{i}\right)=\sum_{i=1}^{n+2}\left(\frac{\mu\left(w_{i}\right)}{\phi\left(w_{i}\right)}-1\right)+n+2
$$

Or

$$
\sum_{i=1}^{n+2}\left(1-\frac{\mu\left(w_{i}\right)}{\phi\left(w_{i}\right)}\right)=2 .
$$

Since the Möbius function $\mu\left(w_{i}\right)$ is either 1,0 , or -1 , and $\phi\left(w_{i}\right)$ is at least 2 , the largest possible $n$ is 2 . So we consider three cases:

$n=0$ : If $x_{1}=\xi$, then $x_{2}=-\xi$. This is case (a).

$n=1$ : If $\mu\left(w_{3}\right) \leq 0$, then we have

$$
\sum_{i=1}^{2}\left(1-\frac{\mu\left(w_{i}\right)}{\phi\left(w_{i}\right)}\right) \leq 1
$$

This implies that

$$
\frac{\mu\left(w_{1}\right)}{\phi\left(w_{1}\right)}=\frac{\mu\left(w_{2}\right)}{\phi\left(w_{2}\right)}=\frac{1}{2} \quad \text { and } \quad \mu\left(w_{3}\right)=0 .
$$

The only possibility is $w_{1}=w_{2}=6$. Thus $x_{1}=\zeta_{6}^{i}, x_{2}=\zeta_{6}^{j}$ where $i, j \in\{1,5\}$, and $x_{3}=\xi$ where the order of $\xi$ is not square-free. As complex numbers,

$$
x_{1}=\frac{1}{2}+a \frac{\sqrt{3}}{2} i \quad \text { and } \quad x_{2}=\frac{1}{2}+b \frac{\sqrt{3}}{2} i,
$$

where $a, b \in\{1,-1\}$. Hence

$$
x_{3}=1-x_{1}-x_{2}=-a \frac{\sqrt{3}}{2} i-b \frac{\sqrt{3}}{2} i,
$$

which is clearly not a root of unity. This yields a contradiction, so $\mu\left(w_{3}\right)=1$. By symmetry, $\mu\left(w_{1}\right)=\mu\left(w_{2}\right)=1$. By (3.2.2), we have

$$
\sum_{i=1}^{3} \frac{1}{\phi\left(w_{i}\right)}=1,
$$

which has three solutions up to permutation: $\left\{\phi\left(w_{1}\right), \phi\left(w_{2}\right), \phi\left(w_{3}\right)\right\}=\{3,3,3\}$, or $\{2,4,4\}$, or $\{2,3,6\}$. But there is no $w$ such that $\phi(w)=3$. Hence $\phi\left(w_{1}\right)=2$ and $\phi\left(w_{2}\right)=\phi\left(w_{3}\right)=4$. Together with $\mu\left(w_{1}\right)=\mu\left(w_{2}\right)=\mu\left(w_{3}\right)=1$, we see that

$$
w_{1}=6 \quad \text { and } \quad w_{2}=w_{3}=10 .
$$

With these constraints, it is straightforward to show that there is no solution to the equation $x_{1}+x_{2}+x_{3}=1$. In conclusion, there is no solution when $n=1$.

$n=2$ : The equation (3.2.2) becomes

$$
\sum_{i=1}^{4} \frac{\mu\left(w_{i}\right)}{\phi\left(w_{i}\right)}=2 .
$$


Since $\phi\left(w_{i}\right) \geq 2$, then the only solution is

$$
\frac{\mu\left(w_{i}\right)}{\phi\left(w_{i}\right)}=\frac{1}{2}
$$

for all $i$. Then $w_{i}=6$ for all $i$. Hence

$$
\left\{x_{1}, x_{2}, x_{3}, x_{4}\right\}=\left\{\zeta_{6}, \zeta_{6}, \zeta_{6}^{5}, \zeta_{6}^{5}\right\}
$$

up to permutations. This is case (b).

Now we can show a part of Theorem 3.1

Proposition 3.3. Suppose $A$ is a quantum polynomial ring of global dimension $n$. If $g \in \operatorname{Aut}_{\mathrm{gr}}(A)$ is a quasi-reflection of finite order, then $g$ is in one of the following cases:

(a) There is a basis of $A_{1},\left\{b_{1}, \cdots, b_{n}\right\}$ such that $g\left(b_{i}\right)=b_{i}$ for all $i \geq 2$ and $g\left(b_{1}\right)=\xi b_{1}$.

(b) The order of $g$ is $2 m$ and there is a basis of $A_{1},\left\{b_{1}, \cdots, b_{n}\right\}$ such that $g\left(b_{i}\right)=b_{i}$ for all $i \geq 3$ and $g\left(b_{1}\right)=\xi b_{1}$ and $g\left(b_{2}\right)=-\xi b_{2}$.

(c) The order of $g$ is 6 and there is a basis of $A_{1},\left\{b_{1}, \cdots, b_{n}\right\}$ such that $g\left(b_{i}\right)=$ $b_{i}$ for all $i \geq 4$ and $g\left(b_{i}\right)=\xi b_{i}$ for $i=1,2$ and $g\left(b_{3}\right)=\bar{\xi} b_{3}$ where $\xi=\zeta_{6}$ or $\zeta_{6}^{5}$.

(d) The order of $g$ is 6 and there is a basis of $A_{1},\left\{b_{1}, \cdots, b_{n}\right\}$ such that $g\left(b_{i}\right)=$ $b_{i}$ for all $i \geq 5$ and $g\left(b_{i}\right)=\zeta_{6} b_{i}$ for $i=1,2$ and $g\left(b_{j}\right)=\zeta_{6}^{5} b_{j}$ for $j=3,4$.

Proof. Note that the Hilbert series of $A$ is $H_{A}(t)=(1-t)^{-n}$. By the definition of a quasi-reflection, $\operatorname{Tr}_{A}(g, t)=(1-t)^{-n+1}(1-\xi t)^{-1}$ for some root of unity $\xi$. By Proposition 1.8 $\xi \neq 1$. Furthermore using equation (1.8.1) we compute $\xi=\operatorname{hdet} g$, so the order of $g$ is a multiple of the order of $\xi$.

Since $g$ has a finite order, there is a basis of $A_{1},\left\{b_{1}, \cdots, b_{n}\right\}$, such that $g\left(b_{i}\right)=$ $x_{i} b_{i}$ for all $i$, where every $x_{i}$ is a root of unity whose order divides the order of $g$.

Since the coefficient of the $t$ term in the power series expansion of $\operatorname{Tr}_{A}(g, t)$ is $\operatorname{tr}\left(\left.g\right|_{A_{1}}\right)$, we have

$$
\sum_{i=1}^{n} x_{i}=(n-1)+\xi
$$

Cancelling all the $x_{i}$ 's with $x_{i}=1$, and permuting $x_{i}$ if necessary, we have

$$
\sum_{i=1}^{m} x_{i}=(m-1)+\xi
$$

where $x_{i} \neq 1$ for all $i=1, \cdots, m$. If $m=1$, this is case (a), and we are done. Now assume $m \geq 2$. First we assume $\xi \neq-1$. Moving $\xi$ to the left-hand side, equation (3.3.1) becomes

$$
\sum_{i=1}^{m} x_{i}-\xi=m-1
$$

Since $m \geq 2$ by Lemma 3.2 there are two possibilities. The first case is Lemma 3.2 case (b): $m-1=2$ and $-\xi=\zeta_{6}$ or $-\xi=\zeta_{6}^{5}$. By symmetry, we may assume $-\xi=\zeta_{6}^{5}$ and $x_{1}=x_{2}=\zeta_{6}, x_{3}=\zeta_{6}^{5}$. This is our case (c).

The second case is $m \geq 2$ and $\xi=-1$. Then equation (3.3.1) becomes

$$
\sum_{i=1}^{m} x_{i}=m-2 .
$$


By Lemma 3.2 there are two cases. Either $m=2, x_{1}=\xi$ and $x_{2}=-\xi$, which is our case (b), or $m-2=2$, which is our case (d).

In the rest of this section we will eliminate most of the cases in Proposition 3.3 (b,c,d). In some cases we will use the notion of a $\mathbb{Z}^{2}$-graded algebra. We say that $R$ is a connected $\mathbb{Z}^{2}$-graded algebra if all the generators of $R$ are either in $\mathbb{Z}^{+} \times \mathbb{Z}^{\geq 0}$ or $\mathbb{Z}^{\geq 0} \times \mathbb{Z}^{+}$. The Hilbert series of a $\mathbb{Z}^{2}$-graded algebra/module $M$ is given by

$$
H_{M}(t, s)=\sum_{i, j} \operatorname{dim} M_{i, j} t^{i} s^{j} .
$$

The usual techniques for Hilbert series of $\mathbb{Z}$-graded algebras/modules extend to the $\mathbb{Z}^{2}$-graded setting. The following lemma is clear.

Lemma 3.4. Let $R$ be a connected $\mathbb{Z}^{2}$-graded algebra.

(a) If we use the assignment $\operatorname{deg}(1,0)=\operatorname{deg}(0,1)=1$ to make $R$ a $\mathbb{Z}$-graded algebra, then $H_{R}(t)=H_{R}(t, t)$.

(b) Suppose that $R$ is noetherian of finite global dimension. Then $H_{A}(t, s)=$ $(e(t, s))^{-1}$, where $e(t, s)$ is an integral polynomial in $t, s$ with $e(0,0)=1$.

(c) Suppose $R$ is noetherian and has finite global dimension. Let $M$ be a finitely generated $\mathbb{Z}^{2}$-graded $R$-module. Then $H_{M}(t, s)=p(t, s) H_{R}(t, s)$ for some integral polynomial $p(t, s)$.

Next we assume that $A$ is generated by $A_{1}$, which has a basis $\left\{b_{1}, \cdots, b_{n}\right\}$.

Proposition 3.5. Suppose that a quantum polynomial algebra $A$ is $\mathbb{Z}^{2}$-graded with $\operatorname{deg} b_{i}=(1,0)$ for all $i=1, \cdots, m$ and $\operatorname{deg} b_{j}=(0,1)$ for all $j=m+1, \cdots, n$. Let $B$ and $C$ be graded subalgebras generated by $\left\{b_{1}, \cdots, b_{m}\right\}$ and $\left\{b_{m+1}, \cdots, b_{n}\right\}$ respectively. Then

(a) $B \cong A /\left(C_{\geq 1}\right)$ and $C \cong A /\left(B_{\geq 1}\right)$.

(b) Both $B$ and $C$ are quantum polynomial rings.

(c) If $m=1$, then $A$ is the Ore extension $C\left[b_{1} ; \sigma\right]$ for some graded algebra automorphism $\sigma$ of $C$. As a consequence, $b_{1}$ is a normal element of $A$.

Proof. For any $\mathbb{Z}^{2}$-graded module $M$, define

$$
M^{\mathbb{Z} \times 0}=\{x \in M \mid \operatorname{deg} x \in \mathbb{Z} \times 0\} .
$$

Similarly we define $M^{0 \times \mathbb{Z}}$. Note that $B=A^{\mathbb{Z} \times 0}$ and $C=A^{0 \times \mathbb{Z}}$. Hence $B$ and $C$ are noetherian. In the following proof, we deal only with $B$. By symmetry, the assertions hold for $C$ also.

(a) There is a natural map $B \rightarrow A \rightarrow A /\left(C_{\geq 1}\right)$. Clearly this is a surjection. For every $x \in B$, $\operatorname{deg} x \in \mathbb{Z} \times 0$. For every $y \in\left(C_{\geq 1}\right)$, deg $y \in \mathbb{Z} \times \mathbb{Z}^{+}$. Hence $B \cap\left(C_{\geq 1}\right)=0$ and the map $B \rightarrow A /\left(C_{\geq 1}\right)$ is injective.

(b) First we prove that $B$ has finite global dimension. Take a graded free resolution of the trivial $A$-module $k$ :

$$
0 \rightarrow P_{n} \rightarrow \cdots \rightarrow P_{1} \rightarrow A \rightarrow k \rightarrow 0
$$

where each $P_{i}$ is a direct sum of $A[-v,-w]$ for some $v, w \geq 0$. Then we have a resolution of $B$-modules:

$$
0 \rightarrow P_{n}^{\mathbb{Z} \times 0} \rightarrow \cdots \rightarrow P_{1}^{\mathbb{Z} \times 0} \rightarrow A^{\mathbb{Z} \times 0} \rightarrow k \rightarrow 0
$$


We claim that $P_{i}^{\mathbb{Z} \times 0}$ is a free $B$-module for every $i$. It suffices to show that each $A[-v,-w]^{\mathbb{Z} \times 0}$ is either 0 or a shift of $B$. It is clear from the definition that

$$
A[-v,-w]^{\mathbb{Z} \times 0}= \begin{cases}0 & w>0 \\ B[-v] & w=0 .\end{cases}
$$

So the trivial $B$-module $k$ has a finite free resolution, and $B$ has finite global dimension. By (a) and [AZ, Corollary 8.4], $B$ satisfies the $\chi$-condition. Since $\underline{\operatorname{Ext}}_{B}^{i}(k, B)_{j}$ is finite dimensional for all $j$, and the $\chi$-condition implies that $\operatorname{Ext}_{B}^{i}(k, B)_{j}$ is bounded, then it follows that $\operatorname{Ext}_{B}^{i}(k, B)$ is finite dimensional. From [Zh, Theorem 1.2] it then follows that $B$ satisfies the Artin-Schelter Gorenstein condition, and hence $B$ is regular. Clearly $B$ is a domain.

Next we study the Hilbert series of $B$. Let $H_{B}(t)=a(t)^{-1}$ and $H_{C}(s)=b(s)^{-1}$. By Lemma 3.4(c), there are $p(t, s)$ and $q(t, s)$ such that $a(t) p(t, s)=e(t, s)$ and $b(s) q(t, s)=e(t, s)$. Setting $t=s$, we have $e(t, t)=(1-t)^{-n}$. Then $a(t)=(1-t)^{a}$ and $b(s)=(1-s)^{b}$ for some integers $a, b$, and $e(t, s)=(1-t)^{a}(1-s)^{b} r(t, s)$. Since $B$ is generated by $m$ elements and $C$ is generated by $n-m$ elements, $a=m$ and $b=n-m$. Thus $e(t, s)=(1-t)^{a}(1-s)^{b}$. Since the resolution (3.5.1) is Koszul, after converting to the $\mathbb{Z}$-grading the resolution (3.5.2) is also Koszul. So the global dimension of $B$ is $m$. Thus we have proved (b).

(c) By (a) with $m=1, C=A /\left(b_{1}\right)$, which has Hilbert series $(1-t)^{-n+1}$. This implies that the Hilbert series of the ideal $\left(b_{1}\right)$ is $t(1-t)^{-n}$. Since $A$ is a domain, the Hilbert series of $b_{1} A$ and $A b_{1}$ are equal to $t(1-t)^{-n}$. Thus $b_{1} A=\left(b_{1}\right)=A b_{1}$, and $b_{1}$ is a normal element of $A$. Since $A /\left(C_{\geq 1}\right)=k\left[b_{1}\right], b_{1}^{2}$ will not appear in any of the relations of $A$. Thus the number of the relations between $b_{1}$ and $b_{j}$ for $j \geq 2$ is $n-1$. The only relations between $b_{1}$ and $b_{j}$ are relations that can be written as, for every $j=2, \cdots, n$,

$$
b_{j} b_{1}=b_{1} \sigma\left(b_{j}\right)
$$

for some $\sigma\left(b_{j}\right) \in C_{1}$. Since $b_{1} C=C b_{1}, \sigma$ extends to an algebra automorphism of $C$. Therefore $A=C\left[b_{1} ; \sigma\right]$.

Lemma 3.6. Let $g$ be a quasi-reflection described in Proposition 3.3(b). Then the order of $g$ is 4 and $\xi=i$.

Proof. We have seen that this situation can occur (Example2.3(b)). If $g$ has order 4 , then this is the only solution up to a permutation. We now assume the order of $g$ is not 4 and produce a contradiction.

Clearly the order of $g$ is not 2. Hence the order of $g$ is at least 6 and the order of $\xi$ is not 4 . If $r:=\sum a_{i j} b_{i} b_{j}=0$ is a relation of $A$, then after applying $g$ we have

$$
\begin{gathered}
g(r):=\sum_{i, j \geq 3} a_{i j} b_{i} b_{j}+\xi^{2}\left(a_{11} b_{1}^{2}+a_{22} b_{2}^{2}\right)-\xi^{2}\left(a_{12} b_{1} b_{2}+a_{21} b_{2} b_{1}\right) \\
+\xi\left(\sum_{i \geq 3}\left(a_{1 i} b_{1} b_{i}+a_{i 1} b_{i} b_{1}\right)\right)-\xi\left(\sum_{i \geq 3}\left(a_{2 i} b_{2} b_{i}+a_{i 2} b_{i} b_{2}\right)\right)=0 .
\end{gathered}
$$


We obtain similar expressions for $g^{p}(r)$ for $p=0,1,2,3,4$, which give rise to a system of equations

$$
\begin{aligned}
& Y_{1}+\left(\xi^{2}\right)^{0} Y_{2}+\left(-\xi^{2}\right)^{0} Y_{3}+\xi^{0} Y_{4}+(-\xi)^{0} Y_{5}=0 \\
& Y_{1}+\left(\xi^{2}\right)^{1} Y_{2}+\left(-\xi^{2}\right)^{1} Y_{3}+\xi^{1} Y_{4}+(-\xi)^{1} Y_{5}=0 \\
& Y_{1}+\left(\xi^{2}\right)^{2} Y_{2}+\left(-\xi^{2}\right)^{2} Y_{3}+\xi^{2} Y_{4}+(-\xi)^{2} Y_{5}=0 \\
& Y_{1}+\left(\xi^{2}\right)^{3} Y_{2}+\left(-\xi^{2}\right)^{3} Y_{3}+\xi^{3} Y_{4}+(-\xi)^{3} Y_{5}=0 \\
& Y_{1}+\left(\xi^{2}\right)^{4} Y_{2}+\left(-\xi^{2}\right)^{4} Y_{3}+\xi^{4} Y_{4}+(-\xi)^{4} Y_{5}=0
\end{aligned}
$$

where $Y_{1}=\sum_{i, j>3} a_{i j} b_{i} b_{j}, Y_{2}=a_{11} b_{1}^{2}+a_{22} b_{2}^{2}, Y_{3}=a_{12} b_{1} b_{2}+a_{21} b_{2} b_{1}, Y_{4}=$ $\sum_{i \geq 3}\left(a_{1 i} b_{1} b_{i}+a_{i 1} b_{i} b_{1}\right)$, and $Y_{5}=\sum_{i \geq 3}\left(a_{2 i} b_{2} b_{i}+a_{i 2} b_{i} b_{2}\right)$. It is easy to check that the determinant of the coefficients in the above system is nonzero when $\xi^{4} \neq 1$. Hence $Y_{i}=0$ for all $i=1,2,3,4,5$. This means that $A$ is $\mathbb{Z}^{2}$-graded when we assign $\operatorname{deg} b_{1}=\operatorname{deg} b_{2}=(1,0)$ and $\operatorname{deg} b_{i}=(0,1)$ for all $i \geq 3$. By Proposition 3.5. the subalgebra $B$ generated by $b_{1}$ and $b_{2}$ is a quantum polynomial ring. So $B$ has only one relation. Let $g^{\prime}$ be the automorphism of $B$ induced by $g$. By Lemma 2.6. $\operatorname{Tr}_{B}(g, t)=p(t) \operatorname{Tr}_{A}(g, t)$. Since $g$ is a quasi-reflection, so is $g^{\prime}$. It suffices to show there is no quasi-reflection $g^{\prime}$ of order larger than 4. The unique relation of $B$ is either $b_{1}^{2}+b_{2}^{2}=0$ or $b_{1} b_{2}+q b_{2} b_{1}=0$ (for $q \neq 0$ ), up to a linear transformation. In both cases, $\operatorname{Tr}_{B}\left(g^{\prime}, t\right)$ is easy to compute:

If $b_{1} b_{2}+q b_{2} b_{1}=0$, then $\operatorname{Tr}_{B}\left(g^{\prime}, t\right)=[(1-\xi t)(1+\xi t)]^{-1}$.

If $b_{1}^{2}+b_{2}^{2}=0$, then $\operatorname{Tr}_{B}\left(g^{\prime}, t\right)=\left(1+\xi^{2} t^{2}\right)^{-1}$.

In each of these cases $g^{\prime}$ is not a quasi-reflection. Therefore the only possibility is that the order of $g$ is 4 .

Lemma 3.7. Let $A$ be a graded domain generated by two elements.

(a) If $A$ has at least one quadratic relation, then $A$ is a quantum polynomial ring; namely, $A$ is isomorphic to either $k_{q}\left[b_{1}, b_{2}\right]$ or $k_{J}\left[b_{1}, b_{2}\right]$.

(b) If $A$ is a quadratic algebra of finite GK-dimension, then $A$ is a quantum polynomial ring.

Proof. (a) Let $r:=\sum_{i, j} a_{i j} b_{i} b_{j}=0$ be one of the relations. Since $A$ is a domain this relation is not a product of two linear terms. Then, possibly after a field extension, $B:=k\left\langle b_{1}, b_{2}\right\rangle /(r)$ is a regular algebra of dimension 2 (see [StZ, p. 1601]), and hence is isomorphic to either $k_{q}\left[b_{1}, b_{2}\right]$ or $k_{J}\left[b_{1}, b_{2}\right]$. In either case, one can check that every homogeneous element in $B$ is a product of linear terms, and thus any proper graded factor ring of $B$ will not be a domain. Therefore $A=B$.

(b) Since $A$ has finite GK-dimension, $A$ cannot be a free algebra. So $A$ has at least one quadratic relation, and the assertion follows from (a).

Lemma 3.8. Let $A$ be a quantum polynomial ring and let $\left\{b_{1}, \cdots, b_{n}\right\}$ be a k-linear basis of $A_{1}$. Suppose $g$ is in $\operatorname{Autgr}_{\mathrm{gr}}(A)$ such that

$$
g\left(b_{1}\right)=\xi b_{1}, g\left(b_{2}\right)=\xi b_{2}, g\left(b_{j}\right)=\xi^{-1} b_{j}, \text { and } g\left(b_{i}\right)=b_{i}
$$

for all $3 \leq j<m$ and $m \leq i$. Suppose $\xi^{4} \neq 1$ and $\xi^{3} \neq 1$. Let $B$ be the subalgebra generated by $b_{1}$ and $b_{2}$. Then $B$ is a quantum polynomial ring and $B \cong A /\left(b_{s}, s \geq 3\right)$. 
Proof. Since $A$ is a quadratic algebra and $\xi^{4} \neq 1$, the relations in $A$ will be homogeneous with respect to the grading

$$
\operatorname{deg}\left(b_{1}\right)=\operatorname{deg}\left(b_{2}\right)=(1,1), \operatorname{deg}\left(b_{j}\right)=(1,-1), \text { and } \operatorname{deg}\left(b_{i}\right)=(1,0),
$$

where $3 \leq j<m$ and $m \leq i$. Hence $A$ is a $\mathbb{Z}^{2}$-graded algebra (different from the one in Lemma 3.4). Any relation in $B$ has degree $(n, n)$, but any relations involving $b_{s}$ for $s \geq 3$ have degree $(n, m)$ for $m<n$. Thus the canonical map

$$
B \rightarrow A \rightarrow A /\left(b_{s} ; s \geq 3\right)
$$

is an isomorphism. Since $B$ is a quadratic domain of finite GK-dimension, by Lemma 3.7 it is a quantum polynomial ring.

Now we are ready to prove Theorem 3.1 .

Proof of Theorem 3.1. It remains to show that there is no quasi-reflection as described in Proposition 3.3 $(\mathrm{c}, \mathrm{d})$. The proofs are very similar for cases (c) and (d), so we work on only case (c).

Suppose that $g$ as described in Proposition 3.3(c) exists. Here $\xi=\zeta_{6}$, and so $\xi^{4} \neq 1$ and $\xi^{3} \neq 1$, and the hypotheses of Lemma 3.8 are satisfied. Thus $B$ is a quantum polynomial ring such that $B=A /\left(b_{s} ; s \geq 3\right)$. When restricted to $B, g$ is equal to $\xi I d_{B}$, and thus $\operatorname{Tr}_{B}(g, t)=(1-\xi t)^{-2}$. By Lemma 2.6.

$$
\frac{1}{(1-\xi t)^{2}}=\operatorname{Tr}_{B}(g, t)=p(t) \operatorname{Tr}_{A}(g, t)=p(t) \frac{1}{(1-t)^{n-1}\left(1-\xi^{\prime} t\right)} .
$$

Since $p(t)$ is a polynomial, we have

$$
p(t)(1-\xi t)^{2}=(1-t)^{n-1}\left(1-\xi^{\prime} t\right),
$$

which is impossible.

If a quasi-reflection is as described in Theorem 3.1(a), then it is like a classical reflection. The quasi-reflection in Theorem 3.1(b) is very mysterious and deserves further study. The following definition seems sensible, at least for quantum polynomial rings.

Definition 3.9. Let $A$ be a quantum polynomial ring.

(a) A quasi-reflection $g$ of $A$ is called a reflection if $\left.g\right|_{A_{1}}$ is a reflection.

(b) A quasi-reflection $g$ of $A$ is called a mystic reflection if $\left.g\right|_{A_{1}}$ is not a reflection.

\section{Mystic REFLECTIONS OF QUANTUM POLYNOMIAL RINGS}

In this section we focus on the mystic reflections of quantum polynomial rings. We will see that all mystic reflections are similar to the automorphism $g$ in Example 2.3 (b).

First we state a lemma that we will use in this analysis; its proof is similar to that of Lemma 3.2, but there are many cases, and some require numerical approximations from Maple, and hence we state it without proof. Let $\zeta_{k}$ be the primitive $k$ th root of unity given by $\zeta_{k}=e^{\frac{2 \pi i}{k}}$.

Lemma 4.1. Consider the system

$$
n=x_{1}+x_{2}+\cdots+x_{n+4},
$$


where $n$ is a nonnegative integer and each $x_{i}$ is a root of unity not equal to 1 . Then $0 \leq n \leq 4$ and the solutions fall into the following cases:

(1) If at least one $x_{i}$ is equal to -1 , then we are in the situation of Lemma 3.2.

(2) If $x_{i}+x_{j}=0$, then again we are in the situation of Lemma 3.2. In particular if $n=0$, then all solutions are of the form $\xi-\xi+\mu-\mu=0$ for roots of unity $\xi$ and $\mu$.

For the remainder suppose that neither (1) nor (2) holds.

(3) If $n=1$, then the solutions are given by

(a) $\left(\zeta_{6}+\zeta_{6}^{5}\right)+\xi\left(1+\zeta_{3}+\zeta_{3}^{2}\right)=1$, where $\xi$ is an arbitrary root of unity;

(b) $\left(\zeta_{10}+\zeta_{10}^{3}+\zeta_{10}^{7}\right)+\left(\zeta_{15}+\zeta_{15}^{11}\right)=1$;

(c) $\left(\zeta_{10}+\zeta_{10}^{3}+\zeta_{10}^{9}\right)+\left(\zeta_{15}^{8}+\zeta_{15}^{13}\right)=1$;

(d) $\left(\zeta_{10}+\zeta_{10}^{7}+\zeta_{10}^{9}\right)+\left(\zeta_{15}^{2}+\zeta_{15}^{7}\right)=1$;

(e) $\left(\zeta_{10}^{3}+\zeta_{10}^{7}+\zeta_{10}^{9}\right)+\left(\zeta_{15}^{4}+\zeta_{15}^{14}\right)=1$.

(4) If $n=2$, then $\left(\zeta_{6}+\zeta_{6}^{5}\right)+\left(\zeta_{10}+\zeta_{10}^{3}+\zeta_{10}^{7}+\zeta_{10}^{9}\right)=2$ is the only solution.

(5) If $n=3$, then there is no solution.

(6) If $n=4$, then $4\left(\zeta_{6}+\zeta_{6}^{5}\right)=4$ is the only solution.

Next we classify the mystic reflections of a quantum polynomial ring.

Lemma 4.2. Let $g$ be a mystic reflection of a quantum polynomial ring A of global dimension $n$. Then the order of $g$ is 4 and

$$
\operatorname{Tr}_{A}(g, t)=\operatorname{Tr}_{A}\left(g^{3}, t\right)=\frac{1}{(1-t)^{n-1}(1+t)}, \quad \operatorname{Tr}_{A}\left(g^{2}, t\right)=\frac{1}{(1-t)^{n-2}(1+t)^{2}} .
$$

Proof. The order of $g$ is 4 by Theorem 3.1(b). By definition,

$$
\operatorname{Tr}_{A}(g, t)=\frac{1}{(1-t)^{n-1}(1-\xi t)} .
$$

From the proof of Proposition 3.3(b), $\xi=-1$, and the formula for $\operatorname{Tr}_{A}(g, t)$ follows. The formula for $\operatorname{Tr}_{A}\left(g^{3}, t\right)$ follows from Lemma 1.4.

Let $\left\{b_{1}, b_{2}, \ldots, b_{n}\right\}$ be a basis for $A_{1}$ as in Theorem 3.1(b), and let $V$ denote the subset $\left\{b_{3}, \ldots, b_{n}\right\}$. From the quadratic term in the Maclaurin series expansion of $\operatorname{Tr}_{A}(g, t)$ we compute $\operatorname{tr}\left(\left.g\right|_{A_{2}}\right)=\left(n^{2}-3 n+4\right) / 2$. We compute $\operatorname{tr}\left(\left.g\right|_{A_{2}}\right)$ directly by first noting that $g\left(b_{1}^{2}\right)=-b_{1}^{2}, g\left(b_{2}^{2}\right)=-b_{2}^{2}, g\left(b_{1} b_{2}\right)=b_{1} b_{2}, g\left(b_{2} b_{1}\right)=b_{2} b_{1}, g\left(b_{1} b_{j}\right)=$ $i b_{1} b_{j}, g\left(b_{j} b_{1}\right)=i b_{j} b_{1}, g\left(b_{2} b_{j}\right)=-i b_{2} b_{j}, g\left(b_{j} b_{2}\right)=-i b_{j} b_{2}$, and $g\left(b_{\ell} b_{j}\right)=b_{\ell} b_{j}$ for $\ell, j \geq 3$. It then follows that

$$
\operatorname{tr}\left(\left.g\right|_{A_{2}}\right)=(-1) \epsilon+i\left|b_{1} V \cup V b_{1}\right|+(-i)\left|b_{2} V \cup V b_{2}\right|+(1) m,
$$

where $\epsilon=\left|\left\{b_{1}^{2}, b_{2}^{2}\right\}\right|$ and $m=\left|\left\{V V \cup b_{1} b_{2} \cup b_{2} b_{1}\right\}\right|$. Hence $\left|b_{1} V \cup V b_{1}\right|=\left|b_{2} V \cup V b_{2}\right|=$ $d \geq n-2$, since $A$ is a domain. From the Hilbert series for the quasi-polynomial ring $A$ we have $\left|A_{2}\right|=\left(n^{2}+n\right) / 2=\epsilon+2 d+m$, so that $m=\left(n^{2}+n\right) / 2-\epsilon-2 d$. Equating the two expressions for $\operatorname{tr}\left(\left.g\right|_{A_{2}}\right)$ gives $\operatorname{tr}\left(\left.g\right|_{A_{2}}\right)=(-1) \epsilon+m=\left(n^{2}-3 n+4\right) / 2$; substituting for $m$ and solving for $d$ gives $d=n-1-\epsilon$, for $\epsilon=1$ or 2 . Since $\epsilon=2$ gives a contradiction, we have $\epsilon=1$ and $d=n-2$. It follows that $b_{1} V=$ $V b_{1}, b_{2} V=V b_{2}$, and $b_{1}^{2}=b_{2}^{2}$, and computing directly we have $\operatorname{tr}\left(\left.g^{2}\right|_{A_{2}}\right)=1-$ $2(n-2)+\left(\left(n^{2}+n\right) / 2-2 n+3\right)=\left(n^{2}-7 n+16\right) / 2$.

We can write $\operatorname{Tr}_{A}\left(g^{2}, t\right)$ as

$$
\operatorname{Tr}_{A}\left(g^{2}, t\right)=\frac{1}{(1-t)^{k}\left(1-x_{1} t\right) \cdots\left(1-x_{n-k} t\right)},
$$


where each $x_{i} \neq 1$ is a root of unity, and for each $x_{i}$ there is an $x_{i^{\prime}}=\overline{x_{i}}$ [Lemma 1.4. By Theorem 3.1 (b), $\operatorname{tr}\left(\left.g^{2}\right|_{A_{1}}\right)=n-4$. Using the Maclaurin series expansion of $\operatorname{Tr}_{A}\left(g^{2}, t\right)$ we have $\operatorname{tr}\left(\left.g^{2}\right|_{A_{1}}\right)=k+x_{1}+\cdots+x_{n-k}$ so that $x_{1}+\cdots+x_{n-k}=(n-k)-4$ with all $x_{i} \neq 1$. We next consider each of the possible solutions for the $x_{i}$ given by Lemma 3.2 and Lemma 4.1, and we compare the quadratic term of each possible trace function to $\left(n^{2}-7 n+16\right) / 2$ to show that the only possibility is the one given in the statement of the theorem.

First we consider the cases where $(n-k)-4$ is negative: i.e., $n-k=0,1,2,3$. When $n-k=0$, then $g^{2}$ is the identity, which it is not. If $n-k=1$, then

$$
\operatorname{Tr}\left(g^{2}, t\right)=\frac{1}{(1-t)^{(n-1)}\left(1-x_{1} t\right)},
$$

a series whose Maclaurin expansion has $t$ coefficient $n-1+x_{1} \neq n-4$. If $n-k=2$, then

$$
\operatorname{Tr}\left(g^{2}, t\right)=\frac{1}{(1-t)^{(n-2)}\left(1-x_{1} t\right)\left(1-x_{2} t\right)},
$$

and the $t$ coefficient in the Maclaurin expansion is $n-2+x_{1}+x_{2}$, which is $n-4$ only if $x_{1}=x_{2}=-1$, giving the $\operatorname{Tr}\left(g^{2}, t\right)$ that is in the statement of the theorem. If $n-k=3$, then the trace is

$$
\operatorname{Tr}\left(g^{2}, t\right)=\frac{1}{(1-t)^{(n-3)}\left(1-x_{1} t\right)\left(1-x_{2} t\right)\left(1-x_{3} t\right)} .
$$

In the Maclaurin expansion of this series the $t$ coefficient is $n-3+x_{1}+x_{2}+x_{3}$; if this coefficient is $n-4$, then we have $-x_{1}-x_{2}-x_{3}=1$, in contradiction to Lemma 3.1 , unless some $x_{i}$ is -1 , in which case the trace is

$$
\operatorname{Tr}\left(g^{2}, t\right)=\frac{1}{(1-t)^{(n-3)}(1+t)\left(1-\zeta^{2} t^{2}\right)} .
$$

This series has Maclaurin expansion with $t^{2}$ coefficient $\left(n^{2}-7 n\right) / 2+\left(7+\zeta^{2}\right)$, which is $\left(n^{2}-7 n\right) / 2+8$ only when $\zeta=-1$, again the form we are trying to prove.

Next suppose that at least one of the $x_{i}=-1$, so without loss of generality we assume $x_{n-k}=-1$. Then

$$
x_{1}+\cdots+x_{n-k-1}=(n-k)-3
$$

and by Lemma 3.2 we have either (a) $n-k-3=0$ and $x_{1}=\zeta$ and $x_{2}=-\zeta$ for $\zeta \neq \pm 1$ a root of unity, and the trace is

$$
\operatorname{Tr}_{A}\left(g^{2}, t\right)=\frac{1}{(1-t)^{(n-3)}(1-\zeta t)(1+\zeta t)(1+t)},
$$

or (b) $n-k-3=2$ and $2\left(\zeta_{6}+\zeta_{6}^{5}\right)=2$ and the trace is

$$
\operatorname{Tr}_{A}\left(g^{2}, t\right)=\frac{1}{(1-t)^{(n-5)}\left(1-\zeta_{6} t\right)^{2}\left(1-\zeta_{6}^{5} t\right)^{2}(1+t)}
$$

In the first case the coefficient of the quadratic term is $\left(n^{2}-7 n\right) / 2+7+\zeta^{2}$, and in the second case it is $\left(n^{2}-7 n\right) / 2+5$. Hence we may assume

$$
x_{1}+x_{2}+\cdots+x_{n-k}=(n-k)-4
$$

and $x_{i} \neq \pm 1$. 
Next suppose that $x_{j}+x_{\ell}=0=\zeta-\zeta$ for some $j, \ell$. This places us again in the situation of Lemma 3.2 and we have either (a) $n-k-4=0$ and $x_{1}=\zeta^{\prime}$ and $x_{2}=-\zeta^{\prime}$, and the trace is

$$
\operatorname{Tr}_{A}\left(g^{2}, t\right)=\frac{1}{(1-t)^{(n-4)}(1-\zeta t)(1+\zeta t)\left(1-\zeta^{\prime} t\right)\left(1+\zeta^{\prime} t\right)},
$$

or (b) $n-k-4=2$ and

$$
\operatorname{Tr}_{A}\left(g^{2}, t\right)=\frac{1}{(1-t)^{(n-6)}\left(1-\zeta_{6} t\right)^{2}\left(1-\zeta_{6}^{5} t\right)^{2}(1-\zeta t)(1+\zeta t)} .
$$

In the first case the coefficient of the quadratic term in the Maclaurin expansion is $\left(n^{2}-7 n\right) / 2+6+\zeta^{2}+\left(\zeta^{\prime}\right)^{2}$ (which is correct only when $\zeta$ and $\zeta^{\prime}$ are \pm 1 , cases already considered), and in the second case it is $\left(n^{2}-7 n\right) / 2+10+\zeta^{2}$ (for which no root of unity provides the correct value).

Next suppose that $n-k=4$, so that

$$
x_{1}+x_{2}+x_{3}+x_{4}=0,
$$

which by multiplying by $x_{1}^{-1}$ reduces to a case handled by Lemma 3.2 and the only solution is

a case handled above.

$$
\zeta-\zeta+\zeta^{\prime}-\zeta^{\prime}=0
$$

Next we suppose that $n-k=5$, and we are in the setting of Lemma 4.1(3) with $x_{1}+\cdots+x_{5}=1$. Then

$$
\operatorname{Tr}_{A}\left(g^{2}, t\right)=\frac{1}{(1-t)^{(n-5)}\left(1-x_{1} t\right)\left(1-x_{2} t\right)\left(1-x_{3} t\right)\left(1-x_{4} t\right)\left(1-x_{5} t\right)},
$$

whose Maclaurin series when $x_{1}+\cdots+x_{5}=1$ begins $1+(n-4) t+\left(n^{2} / 2-7 n / 2+c\right) t^{2}$ where

$$
\begin{gathered}
c=5+x_{1}\left(x_{1}+x_{2}+x_{3}+x_{4}+x_{5}\right)+x_{2}\left(x_{2}+x_{3}+x_{4}+x_{5}\right) \\
\quad+x_{3}\left(x_{3}+x_{4}+x_{5}\right)+x_{4}\left(x_{4}+x_{5}\right)+x_{5}^{2} \\
=5+x_{1}+x_{2}-x_{1} x_{2}+x_{3}-x_{1} x_{3}-x_{2} x_{3}+x_{4}^{2}+x_{4} x_{5}+x_{5}^{2} .
\end{gathered}
$$

We will show that in all five cases $c \neq 8$ so that $\operatorname{Tr}_{A}\left(g^{2}, t\right)$ cannot have $n-k=5$. In case (a), $x_{1}=\zeta_{6}, x_{2}=\zeta_{6}^{5}, x_{3}=\zeta, x_{4}=\zeta \zeta_{3}, x_{5}=\zeta \zeta_{3}^{2}$ for an arbitrary $\zeta$, and we compute that $c=5$. In case (b), $x_{1}=\zeta_{10}, x_{2}=\zeta_{10}^{3}, x_{3}=\zeta_{10}^{7}, x_{4}=\zeta_{15}, x_{5}=\zeta_{15}^{11}$, and we compute $c=5+\zeta_{10}^{3}$. In case (c), $x_{1}=\zeta_{10}, x_{2}=\zeta_{10}^{3}, x_{3}=\zeta_{10}^{9}, x_{4}=\zeta_{15}^{8}, x_{5}=$ $\zeta_{15}^{13}$ and we compute $c=6$. In case $(\mathrm{d}), x_{1}=\zeta_{10}, x_{2}=\zeta_{10}^{7}, x_{3}=\zeta_{10}^{9}, x_{4}=\zeta_{15}^{2}, x_{5}=$ $\zeta_{15}^{7}$ and we compute $c=5+\zeta_{10}$. In case $(\mathrm{e}), x_{1}=\zeta_{10}^{3}, x_{2}=\zeta_{10}^{7}, x_{3}=\zeta_{10}^{9}, x_{4}=$ $\zeta_{15}^{4}, x_{5}=\zeta_{15}^{14}$ and we compute $c=5-\zeta_{10}^{2}$.

Next we suppose that $n-k=6$ and then we are in the setting of Lemma 4.1(4) with $x_{1}+\cdots+x_{6}=2$ and

$$
\operatorname{Tr}_{A}\left(g^{2}, t\right)=\frac{1}{(1-t)^{(n-6)}\left(1-\zeta_{6} t\right)\left(1-\zeta_{6}^{5} t\right)\left(1-\zeta_{10} t\right)\left(1-\zeta_{10}^{3} t\right)\left(1-\zeta_{10}^{7} t\right)\left(1-\zeta_{10}^{9}\right)},
$$

whose Maclaurin series begins $1+(n-4) t+\left(n^{2} / 2-7 n / 2+c\right) t^{2}$ where

$$
\begin{aligned}
c= & 15-6\left(\zeta_{6}+\zeta_{6}^{5}+\zeta_{10}^{3}+\zeta_{10}^{7}+\zeta_{10}^{9}\right)+\left(1+\zeta_{6}^{2}+\zeta_{6}^{4}\right) \\
& \quad+2\left(1-\zeta_{10}+\zeta_{10}^{2}-\zeta_{10}^{3}+\zeta_{10}^{4}\right)+\left(\zeta_{6}+\zeta_{6}^{5}\right)\left(\zeta_{10}+\zeta_{10}^{3}+\zeta_{10}^{7}+\zeta_{10}^{9}\right) \\
= & 15-12+0+0+1=4 \neq 8,
\end{aligned}
$$

so $n-k \neq 6$. 
There is no solution if $n-k=7$ by Lemma 4.1(5), so the last case is $n-k=8$, and

$$
\operatorname{Tr}_{A}\left(g^{2}, t\right)=\frac{1}{(1-t)^{(n-8)}\left(1-\zeta_{6} t\right)^{4}\left(1-\zeta_{6}^{5} t\right)^{4}},
$$

which has Maclaurin series with quadratic coefficient $n^{2} / 2-7 n / 2+2$, so this case is also eliminated.

Hence we have shown that

$$
\operatorname{Tr}_{A}\left(g^{2}, t\right)=\frac{1}{(1-t)^{n-2}(1+t)^{2}}
$$

Here is a partial converse of Theorem 2.4 for mystic reflections.

Proposition 4.3. Let $A$ be a quantum polynomial ring of global dimension $n$ and let $g$ be a mystic reflection.

(a) There is a basis of $A_{1}$, say $\left\{b_{1}, b_{2}, \cdots, b_{n}\right\}$ such that $g\left(b_{1}\right)=i b_{1}, g\left(b_{2}\right)=$ $-i b_{2}$, and $g\left(b_{j}\right)=b_{j}$ for all $j \geq 3$.

(b) $A^{g}$ is regular and $H_{A^{g}}(t)=\left[(1-t)^{n-2}\left(1-t^{2}\right)^{2}\right]^{-1}$.

(c) The subalgebra generated by $b_{1}$ and $b_{2}$ is a quantum polynomial ring subject to one relation $b_{1}^{2}+c b_{2}^{2}=0$ for some nonzero scalar $c$. This subalgebra is also isomorphic to $k_{-1}[x, y]$.

(d) $b_{1}^{2}$ is a normal element of $A$.

Proof. (a) Follows from Theorem 3.1(b) and the definition of mystic reflection.

(b,c,d) For the rest of the proof, let $G$ be the group $\mathbb{Z} /(4)$ and let $k G$ be the group algebra. Define four elements in $k G$ as follows:

$$
\begin{aligned}
& f_{1}=\frac{1}{4}\left(1+g+g^{2}+g^{3}\right), \\
& f_{2}=\frac{1}{4}\left(1-g+g^{2}-g^{3}\right), \\
& f_{3}=\frac{1}{4}\left(1+i g-g^{2}-i g^{3}\right), \\
& f_{4}=\frac{1}{4}\left(1-i g-g^{2}+i g^{3}\right) .
\end{aligned}
$$

It is well known (and easy to check) that $\left\{f_{1}, f_{2}, f_{3}, f_{4}\right\}$ is a complete set of orthogonal idempotents of $k G$. Furthermore,

$$
g f_{1}=f_{1}, \quad g f_{2}=-f_{2}, \quad g f_{3}=-i f_{3}, \quad g f_{4}=i f_{4} .
$$

Since $g$ has order 4 , the eigenvalues of $g$ are $1,-1, i$ and $-i$. Let

$$
\begin{aligned}
& A^{1}=\{x \in A \mid g(x)=x\}=A^{G}, \\
& A^{2}=\{x \in A \mid g(x)=-x\}, \\
& A^{3}=\{x \in A \mid g(x)=-i x\}, \\
& A^{4}=\{x \in A \mid g(x)=i x\} .
\end{aligned}
$$

Then $A=A^{1} \oplus A^{2} \oplus A^{3} \oplus A^{4}$ as $A^{G}$-bimodules. Viewing $f_{j}$ as a projection from $A$ to $f_{j} A$, we see that $A^{j}=f_{j} A$ and the decomposition of $A$ corresponds to the fact that $1=f_{1}+f_{2}+f_{3}+f_{4}$. Since each $f_{j}$ is a projection, we have

$$
H_{A^{j}}(t)=H_{f_{j} A}(t)=\operatorname{Tr}_{A}\left(f_{j}, t\right) .
$$


Since the trace function is additive, we can compute all $\operatorname{Tr}_{A}\left(f_{i}, t\right)$. For example,

$$
\begin{aligned}
\operatorname{Tr}_{A}\left(f_{1}, t\right)= & \frac{1}{4}\left(\sum_{j=0}^{3} \operatorname{Tr}_{A}\left(g^{j}, t\right)\right) \\
= & \frac{1}{4}\left(\frac{1}{(1-t)^{n}}+\frac{1}{(1-t)^{n-1}(1+t)}\right. \\
& \left.\quad+\frac{1}{(1-t)^{n-2}(1+t)^{2}}+\frac{1}{(1-t)^{n-1}(1+t)}\right) \\
= & \frac{1}{4} \frac{(1+t)^{2}+2\left(1-t^{2}\right)+(1-t)^{2}}{(1-t)^{n}(1+t)^{2}} \\
= & \frac{1}{4} \frac{4}{(1-t)^{n}(1+t)^{2}}=\frac{1}{(1-t)^{n}(1+t)^{2}} .
\end{aligned}
$$

The second assertion of (b) follows because

$$
H_{A^{G}}(t)=H_{A^{1}}(t)=\operatorname{Tr}_{A}\left(f_{1}, t\right)=\frac{1}{(1-t)^{n}(1+t)^{2}} .
$$

Similarly we have

$$
\begin{aligned}
& H_{A^{2}}(t)=\operatorname{Tr}_{A}\left(f_{2}, t\right)=\frac{t^{2}}{(1-t)^{n}(1+t)^{2}}, \\
& H_{A^{3}}(t)=\operatorname{Tr}_{A}\left(f_{3}, t\right)=\frac{t}{(1-t)^{n}(1+t)^{2}}, \\
& H_{A^{4}}(t)=\operatorname{Tr}_{A}\left(f_{4}, t\right)=\frac{t}{(1-t)^{n}(1+t)^{2}} .
\end{aligned}
$$

By (a), $b_{1} \in A^{4}$. Since $A$ is a domain,

$$
H_{b_{1} A^{G}}(t)=H_{A^{G} b_{1}}(t)=t H_{A^{G}}=t \frac{1}{(1-t)^{n}(1+t)^{2}}=H_{A^{4}}(t) .
$$

Since $b_{1} A^{G} \subset A^{4}$ and $A^{G} b_{1} \subset A^{4}$, we conclude that

$$
A^{4}=b_{1} A^{G}=A^{G} b_{1} .
$$

In a similar way one can show that

$$
A^{3}=b_{2} A^{G}=A^{G} b_{2}
$$

and

$$
A^{2}=b_{1}^{2} A^{G}=A^{G} b_{1}^{2}=b_{2}^{2} A^{G}=A^{G} b_{2}^{2} .
$$

Therefore $A$ is a free $A^{G}$-module of rank 4 on the left and on the right. By Lemma 1.10(a,c), $A^{G}$ is regular. Thus we have proved (b).

Since both $b_{1}^{2}$ and $b_{2}^{2}$ are in $A^{2}$ and since the dimension of the degree 2 part of $A^{2}$ is $1, b_{1}^{2}$ and $b_{2}^{2}$ are linearly dependent. Since $A$ is a domain, both are nonzero. Thus there is a nonzero scalar $c$ such that $b_{1}^{2}+c b_{2}^{2}=0$. Changing $b_{2}$ by a scalar multiple, we have $b_{1}^{2}-b_{2}^{2}=0$. By Lemma 3.7 (a), the subalgebra generated by $b_{1}$ and $b_{2}$ is a quantum polynomial ring. Clearly $k\left\langle b_{1}, b_{2}\right\rangle /\left(b_{1}^{2}-b_{2}^{2}\right) \cong k_{-1}[x, y]$, so we have proved (c).

Since $b_{1} A^{G}=A^{G} b_{1}$, then $b_{1}^{2} A^{G}=A^{G} b_{1}^{2}$. Since $b_{1}^{2}=b_{2}^{2}$, then $b_{1}^{2}$ commutes with $b_{2}$. Therefore $b_{1}^{2}$ is a normal element in $A$. This is (d). 
Example 4.4. Let $B$ be the quantum algebra generated by $b_{1}$ and $b_{2}$ subject to one relation $b_{1}^{2}-b_{2}^{2}=0$. Let $A$ be the iterated Ore extension of $B, B\left[b_{3} ; \tau\right]\left[b_{4} ; \tau^{\prime}, \delta\right]$, where the automorphism $\tau$ is determined by

$$
\tau\left(b_{1}\right)=-b_{1}, \tau\left(b_{2}\right)=b_{2},
$$

the automorphism $\tau^{\prime}$ is determined by

$$
\tau^{\prime}\left(b_{1}\right)=-b_{1}, \tau^{\prime}\left(b_{2}\right)=b_{2} \quad \text { and } \quad \tau^{\prime}\left(b_{3}\right)=b_{3},
$$

and the $\tau^{\prime}$-derivation $\delta$ is determined by

$$
\delta\left(b_{1}\right)=\delta\left(b_{2}\right)=0 \quad \text { and } \quad \delta\left(b_{3}\right)=b_{1} b_{2}+b_{2} b_{1} .
$$

Then $A$ is a quantum polynomial ring generated by $b_{1}, b_{2}, b_{3}, b_{4}$, subject to the following relations:

$$
\begin{aligned}
b_{1}^{2}-b_{2}^{2} & =0, \\
b_{1} b_{3}+b_{3} b_{1} & =0, \\
b_{2} b_{3}-b_{3} b_{2} & =0, \\
b_{1} b_{4}+b_{4} b_{1} & =0, \\
b_{2} b_{4}-b_{4} b_{2} & =0, \\
b_{3} b_{4}-b_{4} b_{3} & =b_{1} b_{2}+b_{2} b_{1} .
\end{aligned}
$$

Since any graded Ore extension of a regular algebra is regular, $A$ is regular. Also the Ore extension preserves the following properties: being a domain, being noetherian, and having Hilbert series of the form $(1-t)^{-n}$. Thus $A$ is a quantum polynomial ring.

(a) By a direct computation, $A$ does not have a normal element in degree 1 , so there is no normal element in $A_{\geq 1} / A_{\geq 1}^{2}$. But $b_{1}^{2}$ is a normal element of $A$.

(b) Let $g$ be a graded algebra automorphism of $A$ determined by

$$
g\left(b_{1}\right)=i b_{1}, g\left(b_{2}\right)=-i b_{2}, g\left(b_{3}\right)=b_{3}, g\left(b_{4}\right)=b_{4} .
$$

By using a $k$-linear basis of $A$,

$$
\left\{\left(b_{2} b_{1}\right)^{s} b_{2}^{u} b_{3}^{v} b_{4}^{w} \mid s, u, v, w \geq 0\right\} \cup\left\{b_{1}\left(b_{2} b_{1}\right)^{s} b_{2}^{u} b_{3}^{v} b_{4}^{w} \mid s, u, v, w \geq 0\right\},
$$

one can easily verify that

$$
\operatorname{Tr}_{A}(g, t)=\frac{1}{(1-t)^{3}(1+t)}
$$

Therefore $g$ is a mystic reflection.

(c) The fixed subring $C:=A^{g}$ is generated by $b_{3}, b_{4}$ and $z:=b_{1} b_{2}$ subject to the following relations:

$$
\begin{aligned}
z b_{3}+b_{3} z & =0, \\
z b_{4}+b_{4} z & =0, \\
b_{3}^{2} b_{4}-b_{4} b_{3}^{2} & =0, \\
b_{3} b_{4}^{2}-b_{4}^{2} b_{3} & =0 .
\end{aligned}
$$

This algebra is regular of global dimension 4 . Since $z$ is normal in $C$, then there is a normal element in $C_{\geq 1} / C_{\geq 1}^{2}$. 
Remark 4.5. When $A$ is a quantum polynomial ring, we have proved that there is only one kind of mystic reflection: those described in this section. We expect that, when $A$ is a noetherian regular algebra of higher global dimension (but not a quantum polynomial ring), other mystic reflections exist.

\section{A partial Shephard-Todd-Chevalley Theorem}

In this section we prove a simple noncommutative generalization of the ShephardTodd-Chevalley Theorem. The following lemma is a kind of converse of Theorem 3.1(a).

Lemma 5.1. Let $A$ be a quantum polynomial ring with graded algebra automorphism $g$ (not necessarily of finite order). Suppose $\left.g\right|_{A_{1}}$ is a reflection of order not equal to 2. Then:

(a) There is a basis of $A$, say $\left\{b_{1}, \cdots, b_{n}\right\}$, such that $g\left(b_{1}\right)=\xi b_{1}$ and $g\left(b_{j}\right)=b_{j}$ for all $j>1$ and $\xi \neq-1$.

(b) $A=C\left[b_{1} ; \sigma\right]$, where $C$ is a quantum polynomial ring generated by $b_{j}$ for all $j>1$.

(c) $g$ is a quasi-reflection.

(d) $A^{g}$ is regular.

Proof. (a) This is clear by the definition of reflection of $A_{1}$.

(b) Since all the relations of $A$ are quadratic, and the order of $\xi$ is not $2, A$ becomes $\mathbb{Z}^{2}$-graded after we assign $\operatorname{deg} b_{1}=(1,0)$ and $\operatorname{deg} b_{j}=(0,1)$ for all $j \geq 2$. The assertion follows from Proposition 3.5 (c).

(c) Since $g\left(b_{1}\right)=\xi b_{1}$ and $A=C\left[b_{1} ; \sigma\right]=\sum_{i \geq 0} b_{1}^{i} C$,

$$
\operatorname{Tr}_{A}(g, t)=\frac{1}{1-\xi t} \operatorname{Tr}_{C}(g, t)=\frac{1}{(1-t)^{n-1}(1-\xi t)} .
$$

Hence $g$ is a quasi-reflection.

(d) It is clear that $A^{g}=C\left[b_{1}^{w} ; \sigma^{w}\right]$ if the order of $g$ is $w<\infty$, or $A^{g}=C$ if the order of $g$ is infinite.

Lemma 5.2. Let $A$ be a quantum polynomial ring with $\mathrm{GKdim} A>1$ and let $G$ be a finite subgroup of $\operatorname{Autgr}_{\mathrm{gr}}(A)$.

(a) If $G$ contains a quasi-reflection of order not equal to 2 or 4 , then $A \cong C[b ; \sigma]$.

(b) Suppose that $A^{G}$ has finite global dimension (and then $A^{G}$ is regular). If the order of $G$ is odd, then $A \cong C[b ; \sigma]$.

(c) Suppose that $A^{G}$ has finite global dimension (and then $A^{G}$ is regular). If $|G|=4 m$ for some $m>1$ and $G$ does not contain any reflections, then $G$ contains at least 4 mystic reflections.

(d) If $g$ is a reflection of order 2 , then $A^{g}$ is regular and $A$ has a normal element in degree 1.

(e) If $G$ contains a reflection of order 2 , then $A$ has a normal element in degree 1.

Note that a quantum polynomial ring of GKdim $\leq 1$ is either $k$ or $k[x]$. Both of them are commutative and the classical Shephard-Todd-Chevalley theorem applies.

Proof of Lemma [5.2. (a) Let $g$ be a quasi-reflection of order not equal to 2 or 4 . By Theorem 3.1, $g$ is a reflection, namely, $\left.g\right|_{A_{1}}$ is a reflection, and the assertion follows from Lemma 5.1 . 
(b) By Theorem 2.4, $G$ always contains a quasi-reflection $g$. Then the order of $g$ is not 2 or 4 , and the assertion follows from (a).

(c) If $g$ is a mystic reflection, so is $g^{3}$. So the number of mystic reflections is even. Assume there is no reflection and that there are only 2 mystic reflections.

Let $H_{A^{G}}(t)=\left[(1-t)^{n} q(t)\right]^{-1}$ where $q(1) \neq 0$. By Theorem [2.5(b), $\operatorname{deg} q(t)$ is equal to the number of quasi-reflections, which is 2 . Since the roots of $q(t)$ are all roots of unity and the coefficients of $q(t)$ are nonnegative integers, $q(1) \leq 4$. So $|G|=4$, a contradiction.

(d) Let $g$ be a reflection of $A$ of order 2 . So there is a basis of $A_{1}$, say $\left\{b_{1}, \cdots, b_{n}\right\}$ such that $g\left(b_{1}\right)=-b_{1}$ and $g\left(b_{j}\right)=b_{j}$ for all $j \geq 2$.

Let $A^{+}=\{x \in A \mid g(x)=x\}$ and $A^{-}=\{x \in A \mid g(x)=-x\}$. Then $A^{+}=A^{g}$ and $A=A^{+} \oplus A^{-}$as $A^{g}$-bimodules. Since $g$ is a quasi-reflection, $\operatorname{Tr}_{A}(g, t)=$ $\left[(1-t)^{n-1}(1+t)\right]^{-1}$. Using Molien's theorem,

$$
H_{A^{g}}=\frac{1}{2}\left(\frac{1}{(1-t)^{n}}+\frac{1}{(1-t)^{n-1}(1+t)}\right)=\frac{1}{(1-t)^{n-1}\left(1-t^{2}\right)},
$$

and hence

$$
H_{A^{-}}(t)=H_{A}(t)-H_{A^{g}}(t)=\frac{1}{(1-t)^{n}}-\frac{1}{(1-t)^{n-1}\left(1-t^{2}\right)}=\frac{t}{(1-t)^{n-1}\left(1-t^{2}\right)} .
$$

Since $b_{1} \in A^{-}$, both $b_{1} A^{g}$ and $A^{g} b_{1}$ are subspaces of $A^{-}$. Since $A$ is a domain,

$$
H_{b_{1} A^{g}}(t)=H_{A^{g} b_{1}}(t)=\frac{t}{(1-t)^{n-1}\left(1-t^{2}\right)}=H_{A^{-}}(t)
$$

This implies that $b_{1} A^{g}=A^{g} b_{1}=A^{-}$. Recall that $b_{j} \in A^{g}$ for all $j \geq 2$; so $b_{1}$ is normal. Hence $A$ is a free module over $A^{g}$ on both sides. By Lemma 1.10(a,c), $A^{g}$ is regular.

(e) Follows from (d).

Now we are ready to prove Theorem 0.5 .

Theorem 5.3. Let $A$ be a quantum polynomial ring and let $g$ be a graded algebra automorphism of $A$ of finite order.

(a) If $g$ is a quasi-reflection, then the fixed subring $A^{g}$ is regular.

(b) Suppose the order of $g$ is $p^{m}$ for some prime $p$ and some integer $m$. If the fixed subring $A^{g}$ has finite global dimension, then $g$ is a quasi-reflection.

Proof. (a) If $g$ is a mystic reflection, the assertion follows from Proposition 4.3(a). Now let $g$ be a reflection. If the order of $g$ is 2, this follows from Lemma 5.2(d). If the order of $g$ is larger than 2, the assertion follows from Lemma 5.1(d).

(b) Suppose $A^{g}$ is regular. We use induction on $m$. First assume $m=1$. By Theorem 2.4. $G:=\langle g\rangle$ contains a quasi-reflection $g^{i}$ (and hence a reflection since $p \neq 4$ ). Since $p$ is prime, $g$ is a power of $g^{i}$. By Lemma 1.4 $g$ is a quasi-reflection if and only if $g^{i}$ is. So we are done.

Now we assume the order of $g$ is $p^{m}$ for $m \geq 2$. By Theorem 2.4, $G$ contains a quasi-reflection, say $g^{i}$ for some $i$. If $g^{i}$ is a mystic reflection, then the order of $g^{i}$ is 4 , and hence $p=2$. There are at most two elements in $G$ of order 4 . By Lemma 5.2(c), the order of $G$ is 4 . Hence $i=1$ or 3 , and $g$ is a mystic reflection, and hence a quasi-reflection, completing the argument.

As the above paragraph showed, there are at most 2 mystic reflections in $G$ since there are at most two elements of order 4 . Similarly, there is at most one element 
of order 2 in $G$. Further, if $G$ contains a mystic reflection, then the element of order 2 is not a quasi-reflection by Lemma 4.2. If $G$ contains only one quasi-reflection $g$, then $g=g^{-1}$ since $g^{-1}$ is also a quasi-reflection by Lemma 1.4. Thus $|G|=2$ and this case has been taken care of when $m=1$.

Now suppose that we are not in the cases discussed in the above two paragraphs; then $G$ contains a reflection $h$ of order not equal to 2 . Without loss of generality we may write this element as $h:=g^{p^{w}}$ for some $w<m$. So the order of $h$ is $p^{m-w}$. If $w=0$, then we are done. Hence we assume that $w>0$. Let $\left\{b_{1}, \cdots, b_{n}\right\}$ be a basis of $A_{1}$ such that $g\left(b_{j}\right)=\xi_{j} b_{j}$ for all $j$; further let $h\left(b_{1}\right)=\xi b_{1}$ and $h\left(b_{j}\right)=b_{j}$ for all $j>2$. Clearly, $\xi=\xi_{1}^{p^{w}}$. Since the order of $\xi$ is equal to the order of $h$, which is $p^{m-w}$, the order of $\xi_{1}$ is $p^{m}$. By Lemma [5.1 (a,b), $A=C\left[b_{1} ; \sigma\right]$. Let $A^{\prime}=A^{h}=C\left[b_{1}^{p^{m-w}} ; \sigma^{p^{m-w}}\right]$. Then the $G$ action on $A$ induces a $G^{\prime}:=G /(h)$ action on $A^{\prime}$. Reassigning the degree as $\operatorname{deg} b_{1}^{p^{m-w}}=1, A^{\prime}$ is a quantum polynomial ring. It is clear that $A^{G}=A^{\prime G^{\prime}}$. Since $w>0, G^{\prime}$ is generated by $g^{\prime}:=g(h) \in G^{\prime}$ of order $p^{w}$, which is less than $p^{m}$. Since $A^{\prime G^{\prime}}$ is regular, by induction $g^{\prime}$ is a quasi-reflection. Finally we have two cases to deal with. First we assume that $g^{\prime}$ is not a reflection of $A^{\prime}$. Then it is a mystic reflection of $A^{\prime}$. So $p^{w}=2^{2}$. By the choice of $\left\{b_{j}\right\}$ we have

$$
g^{\prime}\left(b_{1}^{2^{m-2}}\right)=g\left(b_{1}^{2^{m-2}}\right)=\xi_{1}^{2^{m-2}} b_{1}^{2^{m-2}}= \pm i b_{1}^{2^{m-2}} .
$$

Without loss of generality we only consider the $+i$ case since the $-i$ case is similar. Up to a permutation we have $g^{\prime}\left(b_{2}\right)=g\left(b_{2}\right)=-i b_{2}$ and $g^{\prime}\left(b_{j}\right)=g\left(b_{j}\right)=b_{j}$ for all $j \geq 3$. By Proposition 4.3( $\mathrm{c}),\left(b_{1}^{2^{m-2}}\right)^{2}=c b_{2}^{2}$ for some nonzero scalar $c$, but this is impossible in $A$. This leaves us the second and the last case: $g^{\prime}$ is a reflection of $A^{\prime}$. By the choice of $\left\{b_{j}\right\}$, we have

$$
g^{\prime}\left(b_{1}^{p^{m-w}}\right)=g\left(b_{1}^{p^{m-w}}\right)=\xi_{1}^{p^{m-w}} b_{1}^{p^{m-w}} \neq b_{1}^{p^{m-w}}
$$

and $g^{\prime}\left(b_{j}\right)=g\left(b_{j}\right)=\xi_{j} b_{j}$ for all $j \geq 2$. By the definition of reflection, we conclude that $\xi_{j}=1$ for all $j \geq 2$. Therefore $g$ is a reflection.

Finally we give an example showing that a reflection of order 2 does exist for some $A$ not isomorphic to $C\left[b_{1} ; \sigma\right]$.

Example 5.4. Let $A$ be the Rees ring of the first Weyl algebra with respect to the standard filtration. So $A$ is generated by $x, y$ and $z$ subject to the relations

$$
x y-y x=z^{2}, \quad z \text { is central. }
$$

Let $g$ be the automorphism of $A$ determined by

$$
g(x)=x, \quad g(y)=y, \quad \text { and } \quad g(z)=-z .
$$

Then $g$ is of order 2. Since $z$ is central, it is easy to check that $\operatorname{Tr}_{A}(g, t)=$ $\left[(1-t)^{2}(1+t)\right]^{-1}$. Hence $g$ is a quasi-reflection and $\left.g\right|_{A_{1}}$ is a reflection. So $g$ is a reflection in the sense of Definition 3.9.

(a) $A^{g}$ is regular by Theorem 5.3 (a).

(b) We claim that $A \not C[b ; \sigma]$. Suppose $A=C[b ; \sigma]$. Then it is easy to check that $z$ (up to a scalar) is the only normal element in degree 1 . Thus $b=z$ and $C=A /(z)$ is commutative. Since $b=z$ is central, then $A$ is commutative, a contradiction. Thus $A \neq C[b ; \sigma]$.

(c) The regular fixed subring $A^{g}$ is generated by $x$ and $y$, and it is isomorphic to $U(L)$, where $L$ is the Lie algebra $k x+k y+k w$, where $w=z^{2}=[x, y]$. Hence the 
fixed subring $A^{g}$ is a regular ring that is different from $A$. We note that $U(L)$ is a two-generator regular ring of dimension 3; hence Proposition 6.4 will show that it does not have any quasi-reflections of finite order, so it is rigid. Hence $U(L)$ can be a fixed subring of a regular ring, but it cannot be the fixed subring of a finite group acting on itself.

We will examine the Rees ring of $A_{n}(k)$ in the next section [Proposition 6.7] and Corollary 6.8.

\section{Rigidity THEOREMS}

In this section we prove the rigidity theorems 0.1 and 0.2 stated in the introduction.

Lemma 6.1. Let $A$ be a noetherian regular algebra. Suppose $A$ has no quasireflection of finite order. Then:

(a) For every finite group $G \subset \operatorname{Aut}_{\mathrm{gr}}(A), A^{G}$ has infinite global dimension.

(b) For every finite group $G \subset \operatorname{Aut}_{\mathrm{gr}}(A), A^{G}$ is not isomorphic to $A$.

Proof. (a) This is Theorem 2.4.

(b) If $A^{G}$ is isomorphic to $A$, then $A^{G}$ has finite global dimension, and so the assertion follows from (a).

Theorem 6.2. Let $A$ be a quantum polynomial ring. Suppose that one of the following conditions holds.

(a) A has no element $b$ of degree 1 such that $b^{2}$ is normal in $A$.

(b) A has no normal element in degree 1, and no subalgebra isomorphic to $k_{-1}\left[b_{1}, b_{2}\right]$.

Then the following conditions hold.

(i) A has no quasi-reflection of finite order.

(ii) For every finite group $G \subset \operatorname{Autgr}_{\mathrm{gr}}(A), A^{G}$ has infinite global dimension.

(iii) For every finite group $G \subset \operatorname{Autgr}(A), A^{G}$ is not isomorphic to $A$.

Proof. By Lemma 6.1 we only need to show (i). So we consider the two cases.

(a) If $A$ has a reflection of finite order, then by Lemmas 5.1(b) and 5.2(e), $A$ has a normal element $b$ in degree 1 . Then $b^{2}$ is normal, a contradiction. If $A$ has a mystic reflection, by Proposition 4.3 (d), $b_{1}^{2}$ is normal, a contradiction. So the assertion (i) follows.

(b) As in case (a), if $A$ has a reflection of finite order, $A$ has a normal element $b$ in degree 1 . This is a contradiction. If $A$ has a mystic reflection, $A$ has a subalgebra isomorphic to $k_{-1}\left[b_{1}, b_{2}\right]$ by Proposition 4.3 (c).

Corollary 6.3. Let $S$ be a non-PI Sklyanin algebra of global dimension $n \geq 3$. Then $S$ has no quasi-reflection of finite order. As a consequence, $S^{G}$ is not regular, and so $S$ is not isomorphic to $S^{G}$, for any nontrivial finite group $G$ of graded algebra automorphisms.

Proof. By Theorem 6.2. it suffices to check that $S$ has no element $b$ in degree 1 such that $b^{2}$ is normal.

Associated to $S$ there is a triple $(E, \sigma, \mathcal{L})$ where $E \subset \mathbb{P}^{n-1}$ is an elliptic curve of degree $n, \mathcal{L}$ is an invertible line bundle over $E$ of degree $n$ and $\sigma$ is an automorphism of $E$ induced by the translation. The basic properties of $S$ can be found in ATV1] for $n=3$, SmS for $n=4$, and TV] for $n \geq 5$. Associated to $(E, \sigma, \mathcal{L})$ one can 
construct the twisted homogeneous coordinate ring, denoted by $B(E, \sigma, \mathcal{L})$. Then there is a canonical surjection

$$
\phi: S \rightarrow B(E, \sigma, \mathcal{L})=: B
$$

such that $\phi$ becomes an isomorphism when restricted to the degree 1 piece. This statement was proved by Tate-Van den Bergh [TV, (4.3)] for $n \geq 5$, by SmithStafford [SmS, Lemma 3.3] for $n=4$ and by Artin-Tate-Van den Bergh ATV1, Section 6] for $n=3$. If $S$ is non-PI, then $\sigma$ has infinite order. Hence $B$ is so-called projectively simple [RRZ, which means that any proper factor ring of $B$ is finite dimensional. Also note that the GK-dimension of $B$ is 2 .

Suppose that there is a $b \in S$ of degree 1 , such that $b^{2}$ is normal. Let $\bar{b}=\phi(b) \in$ $B$. Since $\phi$ is an isomorphism in degree $1, \bar{b} \neq 0$. Now a basic property of $B$ is that it is a domain. Hence $\bar{b}^{2} \neq 0$, and since $b^{2}$ is normal, so is $\bar{b}^{2}$. Therefore $B /\left(\bar{b}^{2}\right)$ is an infinite proper factor ring of $B$, which contradicts the fact that $B$ is projectively simple.

We note that an extensive calculation shows that Corollary 6.3 is also true for 3-dimensional PI Sklyanin algebras, suggesting that the PI hypothesis may not be necessary.

Next we give a class of regular rigid algebras that are not quasi-polynomial rings.

Proposition 6.4. Let $A$ be a noetherian regular algebra of global dimension 3 that is generated by two elements in degree 1. Then A has no quasi-reflection of finite order, and hence no regular fixed subrings $A^{G}$ for $G$ a finite group.

Proof. By the Artin-Schelter classification [AS], the Hilbert series of $A$ is

$$
H_{A}(t)=\frac{1}{(1-t)^{2}\left(1-t^{2}\right)} .
$$

In particular, $A$ has GK-dimension 3 and has two relations of degree 3. Let $g$ be a possible quasi-reflection of $A$ of finite order. Then the trace of $g$ is

$$
\operatorname{Tr}_{A}(g, t)=\frac{1}{(1-t)^{2}\left(1-\xi_{1} t\right)\left(1-\xi_{2} t\right)},
$$

where $\xi_{1}$ and $\xi_{2}$ are roots of unity by Lemma 1.6(d).

Let $\left\{b_{1}, b_{2}\right\}$ be a basis of $A$ such that $g\left(b_{i}\right)=x_{i} b_{i}$ for $i=1,2$, where $x_{1}$ and $x_{2}$ are roots of unity. Comparing the coefficients of $t$ in the Maclaurin series expansion of $\operatorname{Tr}_{A}(g, t)$, we obtain that

$$
\operatorname{tr}\left(\left.g\right|_{A_{1}}\right)=x_{1}+x_{2}=1+1+\xi_{1}+\xi_{2} .
$$

By Lemma 3.2 there are three solutions:

Solution 1: $\xi_{1}=\xi_{2}=-1, x_{1}=-x_{2}$.

Solution 2: $\xi_{1}=-1, x_{1}=1, \xi_{2}=x_{2}$ up to a permutation.

Solution 3: $\left\{x_{1}, x_{2},-\xi_{1},-\xi_{2}\right\}=\left\{\zeta_{6}, \zeta_{6}, \zeta_{6}^{5}, \zeta_{6}^{5}\right\}$ up to a permutation.

Next we show that each of these is impossible.

Solution 1: Since $\xi_{1}=\xi_{2}=-1, \operatorname{tr}\left(\left.g\right|_{A_{2}}\right)=2$. The eigenvalues of $\left.g\right|_{A_{2}}$ are $x_{1}^{2}$ with eigenspace $k b_{1}^{2}+k b_{2}^{2}$ and $-x_{1}^{2}$ with eigenspace $k b_{1} b_{2}+k b_{2} b_{1}$. So $\operatorname{tr}\left(\left.g\right|_{A_{2}}\right)=0$, a contradiction.

Solution 2: Since $\xi_{1}=-1$ and $\xi_{2}=x_{2}, \operatorname{tr}\left(\left.g\right|_{A_{2}}\right)=2+x_{2}+x_{2}^{2}$. Applying $g$ to the space $A_{2}$, we see that $\operatorname{tr}\left(\left.g\right|_{A_{2}}\right)=1+2 x_{2}+x_{2}^{2}$. Hence $x_{2}=1$. This is impossible since $g$ is not the identity. 
Solution 3: If $x_{1}=x_{2}$, then $\operatorname{Tr}_{A}(g, t)=H_{A}\left(x_{1} t\right)$, which shows that $g$ is not a quasi-reflection. Hence $x_{1} \neq x_{2}$. Up to a permutation we may assume $x_{1}=-\xi_{1}=$ $\zeta_{6}$ and $x_{2}=-\xi_{2}=\zeta_{6}^{5}$. Expanding $\operatorname{Tr}_{A}(g, t)$, we have

$$
\operatorname{Tr}_{A}(g, t)=\frac{1}{(1-t)^{2}\left(1+\zeta_{6} t\right)\left(1+\zeta_{6}^{5} t\right)}=1+t+t^{2}+2 t^{3}+\cdots .
$$

Consequently, $\operatorname{tr}\left(\left.g\right|_{A_{3}}\right)=2$. Now consider $\left.g\right|_{A_{3}}$. The eigenvalues of $\left.g\right|_{A_{3}}$ are either $-1\left(=\zeta_{6}^{3}=\left(\zeta_{6}^{5}\right)^{3}\right), \zeta_{6}$ or $\zeta_{6}^{5}$. So we have

$$
2=\operatorname{tr}\left(\left.g\right|_{A_{3}}\right)=n_{1}(-1)+n_{2} \zeta_{6}+n_{3} \zeta_{6}^{5}, \quad n_{1}, n_{2}, n_{3} \geq 0,
$$

where $n_{1}+n_{2}+n_{3}=6$ is the $\operatorname{dim} A_{3}$. But this is impossible.

Proposition 6.4 applies to a noetherian graded down-up algebra $A=A(\alpha, \beta, 0)$, where $\beta \neq 0$ (see $[\mathrm{BR}, \mathrm{KMP}]$ ). This algebra is generated by $d, u$ subject to the two relations:

$$
d u^{2}=\alpha u d u+\beta u^{2} d \quad \text { and } \quad d^{2} u=\alpha d u d+\beta u d^{2} .
$$

It is a noetherian regular algebra of global dimension 3 , and so by the above proposition, $A$ has no quasi-reflection of finite order.

Let $\mathfrak{g}$ be a Lie algebra, finite dimensional over $k$ with Lie bracket [, ]. Let $\left\{b_{1}, \cdots, b_{n}\right\}$ be a $k$-linear basis of $\mathfrak{g}$. The homogenization of $U(\mathfrak{g})$, denoted by $H(\mathfrak{g})$, is defined to be its Rees ring with respect to the standard filtration of $U(\mathfrak{g})$. It is a connected graded algebra generated by the vector space $\mathfrak{g}+k z$ subject to the relations

$$
b_{i} z=z b_{i} \quad \text { and } \quad b_{i} b_{j}-b_{j} b_{i}=\left[b_{i}, b_{j}\right] z
$$

for all $i, j$. To distinguish it from the Lie product, we use $\lfloor x, y\rfloor$ to denote $x y-y x$ in an algebra. Then the relations of $H(\mathfrak{g})$ can be written as

$$
\left\lfloor b_{i}, z\right\rfloor=0 \quad \text { and }\left\lfloor b_{i}, b_{j}\right\rfloor=\left[b_{i}, b_{j}\right] z .
$$

It is well known that $H(\mathfrak{g})$ is a quantum polynomial ring of dimension $n+1$ Sm2, §12]. By definition, $z$ is a central element such that $H(\mathfrak{g}) /(z-1) \cong U(\mathfrak{g})$ and $H(\mathfrak{g}) /(z) \cong k[\mathfrak{g}]$.

Lemma 6.5. Let $\mathfrak{g}$ be a finite dimensional Lie algebra with no 1-dimensional Lie ideal, and let $H=H(\mathfrak{g})$. Then:

(a) Up to a scalar, $z$ is the only nonzero normal element of $H$ in degree 1 .

(b) Up to a scalar, $z$ is the only normal element in $H-k$ such that $H /(z) \cong k[\mathfrak{g}]$.

(c) $H \neq C[b ; \sigma]$ as graded rings.

(d) $H$ does not have any quasi-reflection of finite order.

(e) Suppose that $\mathfrak{g}^{\prime}$ is another Lie algebra with no 1-dimensional Lie ideal. If $H \cong H\left(\mathfrak{g}^{\prime}\right)$ as ungraded algebras, then $\mathfrak{g} \cong \mathfrak{g}^{\prime}$ as Lie algebras.

Proof. (a) Suppose there is another normal element in degree 1. We may write it as $b+\xi z$ for some $0 \neq b \in \mathfrak{g}$ and some $\xi \in k$. Since $b+\xi z$ is normal, for every $0 \neq x \in \mathfrak{g}$, there are elements $y \in \mathfrak{g}$ and $\xi^{\prime} \in k$ such that

$$
x(b+\xi z)=(b+\xi z)\left(y+\xi^{\prime} z\right) .
$$

Modulo $z$ we have $x b=b y$ in $k[\mathfrak{g}$ ], and hence $y=x$. Thus (6.5.1) implies that

$$
(b+\xi z) \xi^{\prime} z=\lfloor x, b+\xi z\rfloor=\lfloor x, b\rfloor=[x, b] z .
$$

This implies that $[x, b]=\xi^{\prime} b$. Since $x$ is arbitrary, $k b$ is a 1-dimensional Lie ideal. This yields a contradiction. 
(b) Let $w \in H-k$ be another normal element in $H$ such that $H /(w) \cong k[\mathfrak{g}]$. Then $\lfloor H, H\rfloor \subset w H=H w$. Consequently,

$$
[\mathfrak{g}, \mathfrak{g}] z=\lfloor\mathfrak{g}, \mathfrak{g}\rfloor \subset w H .
$$

Since $\mathfrak{g}$ has no 1-dimensional Lie ideal, the Lie ideal $[\mathfrak{g}, \mathfrak{g}]$ must have dimension at least 2. Picking two linearly independent elements $b_{1}, b_{2} \in[\mathfrak{g}, \mathfrak{g}]$, we have $b_{1} z, b_{2} z \in$ $w H$. Write $b_{1} z=c_{1} w$ and $b_{2} z=c_{2} w$. Since $H$ is a domain, $\operatorname{deg} c_{i}+\operatorname{deg} w=$ $\operatorname{deg} b_{1} z=2$. Since $b_{1}$ and $b_{2}$ are linearly independent, the degree of $w$ cannot be 2 . Hence $\operatorname{deg} w=1$. A simple calculation shows that $w=z$ up to a scalar.

(c) By (a), $z$ is the only normal element in degree 1 . If $H \cong C[b ; \sigma]$, then $b$ must be $z$ and $\sigma=I d_{C}$. In this case $C=H /(b)=H /(z)$, which is isomorphic to the commutative polynomial ring. Therefore $H \cong C[b ; \sigma]$ is commutative, a contradiction.

(d) Suppose $g$ is a quasi-reflection. If $g$ is a reflection of order larger than 2, by Lemma 5.1 (b), $H \cong C[b ; \sigma]$. This is impossible by (c).

If $g$ is a reflection of order 2, by the proof of Lemma $5.2(\mathrm{~d})$, there is a basis of $H_{1},\left\{b, c_{1}, \cdots, c_{n}\right\}$, so that $b$ is a normal element of $H$ and $g(b)=-b$ and $g\left(c_{i}\right)=c_{i}$ for all $i$. By (a), $z$ is the only normal element in degree 1 . Hence

$$
b=z, \quad c_{i}=b_{i}+\xi_{i} z
$$

for a basis $\left\{b_{i}\right\}$ of $\mathfrak{g}$ and for some $\xi_{i} \in k$. Now we compute $g\left(\left\lfloor c_{i}, c_{j}\right\rfloor\right)$ in two ways:

$$
g\left(\left\lfloor c_{i}, c_{j}\right\rfloor\right)=\left\lfloor g\left(c_{i}\right), g\left(c_{j}\right)\right\rfloor=\left\lfloor c_{i}, c_{j}\right\rfloor=\left\lfloor b_{i}+\xi_{i} z, b_{j}+\xi_{j} z\right\rfloor=\left\lfloor b_{i}, b_{j}\right\rfloor=\left[b_{i}, b_{j}\right] z
$$

and

$$
g\left(\left\lfloor c_{i}, c_{j}\right\rfloor\right)=g\left(\left[b_{i}, b_{j}\right] z\right)=g\left(\left[b_{i}, b_{j}\right]\right) g(z)=\left(\left[b_{i}, b_{j}\right]+\xi z\right)(-z)
$$

for some $\xi \in k$. The only possible solution is $\xi=0$ and $\left[b_{i}, b_{j}\right]=0$. But we can choose $i, j$ such that $\left[b_{i}, b_{j}\right] \neq 0$, which yields a contradiction.

Finally if $g$ is a mystic reflection (of order 4), then there are two linearly independent elements $c_{1}$ and $c_{2}$ in $H_{1}$ such that $c_{1}^{2}=c_{2}^{2}$ [Proposition 4.3(c)]. Since $H /(z)$ is a commutative polynomial ring, $c_{1}= \pm c_{2}$ in $H /(z)$. Up to a scalar, we may assume $c_{1}=b+z$ and $c_{2}=b+\tau z$ where $b$ is a nonzero element in $\mathfrak{g}$ and $1 \neq \tau \in k$. In this form, one can easily check that $c_{1}^{2} \neq c_{2}^{2}$ in $H$. Therefore $H$ has no mystic reflection.

(e) Let $H^{\prime}=H\left(\mathfrak{g}^{\prime}\right)$. Let $f: H \rightarrow H^{\prime}$ be an isomorphism of (ungraded) algebras. By (b), $f(z)=\xi z$ for some nonzero scalar $\xi$. There is an automorphism of the graded algebra $H^{\prime}$ sending $\xi z$ to $z$. So we can assume that $f(z)=z$.

Let $\left\{b_{1}, \cdots, b_{n}\right\}$ be a basis of $\mathfrak{g}$. For every $j$, write $f\left(b_{j}\right)=\xi_{j}+\sigma\left(b_{j}\right)$ where $\xi_{j} \in k$ and $\sigma\left(b_{j}\right) \in H_{\geq 1}^{\prime}$. We claim that $z \mapsto z:=\sigma(z), b_{j} \mapsto \sigma\left(b_{j}\right)$ defines an isomorphism from $H$ to $H^{\prime}$. First we show that $\sigma$ defines an algebra homomorphism; namely, $\sigma$ preserves the defining relations. Recall that the defining relations of $H$ are

$$
\left\lfloor b_{j}, z\right\rfloor=0 \quad \text { and }\left\lfloor b_{j}, b_{f}\right\rfloor=\left[b_{j}, b_{f}\right\rfloor z .
$$

Since $\sigma(z)=z$ is central in $H^{\prime}$, we have $\left\lfloor\sigma\left(b_{j}\right), z\right\rfloor=0$, namely, $\sigma$ preserves the first set of relations. Applying $f$ to the second set of relations, we have

$$
\left\lfloor f\left(b_{j}\right), f\left(b_{f}\right)\right\rfloor=f\left(\left[b_{j}, b_{f}\right]\right) f(z)=f\left(\left[b_{j}, b_{f}\right]\right) z .
$$


Since $\left\lfloor H^{\prime}, H^{\prime}\right\rfloor \subset z \mathfrak{g}^{\prime} H^{\prime}, f\left(\left[b_{j}, b_{f}\right]\right) \in \mathfrak{g}^{\prime} H^{\prime}$. Hence $\sigma\left(\left[b_{j}, b_{f}\right]\right)=f\left(\left[b_{j}, b_{f}\right]\right)$ after extending $\sigma$ linearly. Now

$$
\begin{aligned}
\left\lfloor\sigma\left(b_{j}\right), \sigma\left(b_{f}\right)\right\rfloor & =\left\lfloor f\left(b_{j}\right)-\xi_{j}, f\left(b_{f}\right)-\xi_{f}\right\rfloor \\
& =\left\lfloor f\left(b_{j}\right), f\left(b_{f}\right)\right\rfloor=f\left(\left[b_{j}, b_{f}\right]\right) z=\sigma\left(\left[b_{j}, b_{f}\right]\right) \sigma(z) .
\end{aligned}
$$

Therefore $\sigma$ preserves the second set of defining relations. Thus we have proved that $\sigma$ is an algebra homomorphism. Since $\left\{b_{1}, \cdots, b_{n}, z\right\}$ generates $H$ and $f$ is an isomorphism, then $\left\{f\left(b_{1}\right), \cdots, f\left(b_{n}\right), z\right\}$ generates $H^{\prime}$. Hence $\left\{\sigma\left(b_{1}\right), \cdots, \sigma\left(b_{n}\right), z\right\}$ generates $H^{\prime}$ also, and we have shown that $\sigma$ is an algebra isomorphism from $H$ to $H^{\prime}$.

Note that $\sigma\left(H_{\geq 1}\right) \subset H_{\geq 1}^{\prime}$. Since $\sigma$ is an isomorphism, $\sigma\left(H_{\geq 1}\right)=H_{\geq 1}^{\prime}$. Since $H$ is generated in degree 1 , it has a natural filtration

$$
\left\{F_{-j} H:=\left(H_{\geq 1}\right)^{j}=H_{\geq j} \mid j \in \mathbb{Z}\right\} .
$$

The same is true for $H^{\prime}$. Thus $\sigma$ is a filtered isomorphism that induces a graded algebra isomorphism $\tau:=\operatorname{gr} \sigma: \operatorname{gr} H \rightarrow \operatorname{gr} H^{\prime}$. Since gr $H=H, \tau$ is a graded isomorphism from $H$ to $H^{\prime}$ sending $z$ to $z$.

For every $b \in \mathfrak{g}$ write $\tau(b)=\phi(b)+\chi(b) z$ where $\phi(b) \in \mathfrak{g}^{\prime}$ and $\chi$ is a linear map from $\mathfrak{g}$ to $k$. We claim that $\phi: \mathfrak{g} \rightarrow \mathfrak{g}^{\prime}$ is a Lie algebra isomorphism. Since $\tau(z)=z$, $\phi$ is an isomorphism of $k$-vector spaces. To show $\phi$ preserves the Lie product, we use the following direct computation:

$$
\begin{aligned}
\phi\left(\left[b_{j}, b_{f}\right]\right) z & =\tau\left(\left[b_{j}, b_{f}\right]\right) z-\chi\left(\left[b_{j}, b_{f}\right]\right) z^{2}=\tau\left(\left[b_{j}, b_{f}\right] z\right)-\xi z^{2} \\
& =\tau\left(\left\lfloor b_{j}, b_{f}\right\rfloor\right)-\xi z^{2}=\left\lfloor\tau\left(b_{j}\right), \tau\left(b_{f}\right)\right\rfloor-\xi z^{2} \\
& =\left\lfloor\phi\left(b_{j}\right)+\chi\left(b_{j}\right) z, \phi\left(b_{f}\right)+\chi\left(b_{f}\right) z\right\rfloor-\xi z^{2} \\
& =\left\lfloor\phi\left(b_{j}\right), \phi\left(b_{f}\right)\right\rfloor-\xi z^{2}=\left[\phi\left(b_{j}\right), \phi\left(b_{f}\right)\right] z-\xi z^{2} .
\end{aligned}
$$

Thus $\phi\left(\left[b_{j}, b_{f}\right]\right)=\left[\phi\left(b_{j}\right), \phi\left(b_{f}\right)\right]$ and $\xi:=\chi\left(\left[b_{j}, b_{f}\right]\right)=0$. Therefore $\phi$ is a Lie algebra isomorphism from $\mathfrak{g}$ to $\mathfrak{g}^{\prime}$.

Proof of Theorem 0.2 (a). By Lemma 6.5(d), $H:=H(\mathfrak{g})$ does not have any quasireflections of finite order. By Theorem 2.4 for any finite group $G \subset \operatorname{Aut}_{\mathrm{gr}}(H), H^{G}$ does not have finite global dimension. Thus $H^{G} \cong H(\mathfrak{g})$ implies that $G$ is trivial. Since $G=\{1\}$, then $H(\mathfrak{g}) \cong H\left(\mathfrak{g}^{\prime}\right)$, which implies $\mathfrak{g} \cong \mathfrak{g}^{\prime}$ by Lemma 6.5(e).

Example 6.6. This example shows that the condition about nonexistence of 1dimensional Lie ideals in Theorem 0.2 is necessary.

Let $\mathfrak{g}$ be the 2-dimensional solvable Lie algebra $k x+k y$ with $[x, y]=y$. Then $k y$ is a 1-dimensional Lie ideal. The homogenization $H(\mathfrak{g})$ of $U(\mathfrak{g})$ is generated by $x, y, z$ subject to the following relations:

$$
x y-y x=y z, \quad z x=x z, \quad z y=y z .
$$

It is easy to see that $H(\mathfrak{g})$ is isomorphic to an Ore extension $k[x, z][y ; \sigma]$, where $\sigma(x)=x+z$ and $\sigma(z)=z$. Let $g$ be an automorphism of $H(\mathfrak{g})$ determined by

$$
g(x)=x, g(z)=z \quad \text { and } \quad g(y)=-y .
$$

It is easy to see that $g$ is a reflection of $H(\mathfrak{g})$. The fixed subring of $H(\mathfrak{g})$ is isomorphic to $k[x, z]\left[y^{2} ; \sigma^{2}\right]$. There is an isomorphism $\phi: H(\mathfrak{g}) \rightarrow H(\mathfrak{g})^{g}$ defined by

$$
\phi: x \mapsto x, \quad y \mapsto y^{2}, \quad z \mapsto 2 z .
$$


Finally let us consider the proof of Theorem $0.2(\mathrm{~b})$. Let $A$ be the Rees ring of the Weyl algebra $A_{n}(k)$ with respect to the standard filtration; then $A$ is the algebra with generating set $\left\{x_{i}, y_{i}, z: i=1,2, \ldots, n\right\}$ subject to the relations $x_{i} y_{i}-y_{i} x_{i}=z^{2}$ for $i=1,2, \ldots, n$, and with all other generators commuting. The algebra $A$ is a regular domain of dimension $2 n+1$ [Le, 3.6] with Hilbert series $H_{A}(t)=1 /(1-t)^{2 n+1}$. We first find the reflection groups of $A$.

Proposition 6.7. Let $A$ be the Rees ring of the Weyl algebra $A_{n}(k)$.

(a) If $g$ is a quasi-reflection of $A$, then $g$ is a reflection of the form $g\left(x_{i}\right)=$ $x_{i}+a_{i} z, g\left(y_{i}\right)=y_{i}+b_{i} z$, and $g(z)=-z$ for elements $a_{i}, b_{i} \in k$.

(b) If $G$ is a finite group of graded automorphisms of $A$ such that $A^{G}$ is regular, then $G=\{I d, g\}$ for a reflection $g$.

Proof. (a) Let $g$ be a quasi-reflection of $A$. Then

$$
\operatorname{Tr}(g, t)=\frac{1}{(1-t)^{2 n}(1-\xi t)}
$$

for some scalar $\xi$. Since $z$ is the only central element of degree 1, we must have that $g(z)=\lambda z$ for some scalar $\lambda$.

Suppose that $\lambda \neq 1$. Since $\langle z\rangle$ is $g$-invariant, $g$ induces an automorphism $\bar{g}$ of $\bar{A}=A /\langle z\rangle$. Since $\operatorname{Tr}_{\bar{A}}(\bar{g}, t)=(1-\lambda t) \operatorname{Tr}_{A}(g, t)=\left(e_{\bar{g}}(t)\right)^{-1}$, we have that $\operatorname{Tr}_{\bar{A}}(\bar{g}, t)=(1-t)^{-2 n}$, and $\bar{g}$ must be the identity on $\bar{A}=k\left[x_{i}, y_{i}, i=1,2, \ldots, n\right]$. Then $g\left(x_{i}\right)=x_{i}+a_{i} z, g\left(y_{i}\right)=y_{i}+b_{i} z$, and $g(z)=\lambda z$. In order that the relations of $A$ are preserved by $g$ it follows that $\lambda^{2}=1$, so that $g$ has the form stated.

Now suppose that $g(z)=z$. If $g$ is a reflection, then by Lemma 5.1 (b) and the proof of Lemma 5.2 (d) there is a basis $\left\{b_{1}, b_{2}, \ldots, b_{2 n+1}\right\}$ of $A_{1}$ such that $g\left(b_{1}\right)=$ $\xi b_{1}, g\left(b_{i}\right)=b_{i}$ for $i \geq 2$, and $b_{1}$ is a normal element of $A$. Since there are no normal elements in $A_{1}$ other than multiples of $z$, this cannot be, and hence there are no reflections with $g(z)=z$.

Now suppose that $g$ is a mystic reflection with $g(z)=z$. Then by Proposition 4.2 there is a basis $\left\{b_{1}, b_{2}, \ldots, b_{2 n+1}\right\}$ of $A_{1}$ such that $g\left(b_{1}\right)=i b_{1}, g\left(b_{2}\right)=-i b_{2}$, $g\left(b_{i}\right)=b_{i}$ for $i \geq 3$, and $b_{1}^{2}$ is a normal element of $A$. Since multiples of $z$ are the only elements of $A_{1}$ which square to normal elements, we have shown that there are no mystic reflections of $A$. Hence (a) follows.

(b) Suppose that $G$ is a finite group of graded automorphisms of $A$ such that $A^{G}$ is regular. Then $G$ must contain a quasi-reflection $g_{1}$ by Theorem 2.4 Suppose that $G$ contains another quasi-reflection $g_{2} \neq g_{1}$. By (a) these quasi-reflections are reflections that can be represented on $A_{1}$ by matrices

$$
M_{g_{1}}=\left[\begin{array}{cc}
I & \overline{0} \\
\bar{v} & -1
\end{array}\right], M_{g_{2}}=\left[\begin{array}{cc}
I & \overline{0} \\
\bar{u} & -1
\end{array}\right]
$$

where $I$ is a $2 n \times 2 n$ identity matrix and $\bar{u} \neq \bar{v}$. Then $g_{1} g_{2}$ is represented by the product matrix

$$
\left[\begin{array}{cc}
I & \overline{0} \\
\bar{v}-\bar{u} & +1
\end{array}\right]
$$

which has infinite order. Hence $G$ can contain exactly one quasi-reflection. Since $A^{G}$ is regular, its Hilbert series has the form

$$
H_{A^{G}}(t)=\frac{1}{(1-t)^{2 n+1} q(t)},
$$


where $q(t)$ is a product of cyclotomic polynomials. By Theorem 2.5 (b), $\operatorname{deg} q(t)$ is the number of quasi-reflections in $G$, and hence must be 1 . Consequently $q(t)=$ $1+t$. Also by Theorem $2.4(\mathrm{~b}), q(1)=2=|G|$. Thus $G=\{I d, g\}$ for a reflection $g$.

Note that $A^{g}$ is regular by Theorem 5.3 (a).

Corollary 6.8. Let $A$ be the Rees ring of the Weyl algebra $A_{n}(k)$. Then $A$ is not isomorphic (as an ungraded algebra) to $A^{G}$ for any finite group of graded automorphisms.

Proof. If $A^{g}$ has infinite global dimension, then $A^{g} \approx A$.

If $A^{g}$ has finite global dimension, by Proposition 6.7(b), $G=\{I d, g\}$ for a reflection $g$ whose matrix on $A_{1}$ is of the form

$$
\left[\begin{array}{cc}
I & \overline{0} \\
\bar{v} & -1
\end{array}\right]
$$

for $\bar{v}=\left[a_{1}, b_{1}, \ldots, a_{n}, b_{n}\right]$. A computation shows that if $X_{i}=x_{i}+\frac{a_{i}}{2} z$ and $Y_{i}=$ $y_{i}+\frac{b_{i}}{2} z$ for $i=1,2, \ldots, n$, then $A^{g}$ is generated by the set

$$
\left\{X_{1}, Y_{1}, X_{2}, Y_{2}, \ldots, X_{n}, Y_{n}, z^{2}\right\}
$$

subject to the relations $X_{i} Y_{i}-Y_{i} X_{i}=z^{2}$, with all other generators commuting. In particular, $A^{g}$ is generated by $2 n$ elements since $z^{2}=X_{i} Y_{i}-Y_{i} X_{i}$. But $A$ is (minimally) generated by $2 n+1$ elements. Therefore $A^{g} \neq A$.

\section{Further Questions}

The results we have obtained suggest that the invariant theory of Artin-Schelter regular rings merits further study. We conclude by describing a few directions that seem particularly interesting.

Bi-reflections. In the case that $A=k\left[x_{1}, \cdots, x_{n}\right]$ Kac and Watanabe [KW] and Gordeev $\mathrm{Go}$ independently proved that if $A^{G}$ is a complete intersection and $G$ is a finite subgroup of $G L_{n}(k)$, then $G$ is generated by bi-reflections (elements such that $\operatorname{rank}(g-I) \leq 2)$. Following our generalization of reflections, a natural generalization of bi-reflections to a regular algebra $A$ of dimension $n$ is to call a graded automorphism $g$ of $A$ a quasi-bi-reflection if its trace has the form

$$
\operatorname{Tr}_{A}(g, t)=\frac{1}{(1-t)^{n-2} q(t)}
$$

where $n$ is the GK-dimension of $A$ and $q(1) \neq 1$. We have constructed some examples that suggest that this is a reasonable definition (the fixed ring is a commutative complete intersection). As in the case of reflections, there are "mystic quasi-bireflections" (quasi-bi-reflections that are not bi-reflections of $A_{1}$ ). The notion of bi-reflection may be useful in determining the proper notion of a noncommutative complete intersection.

Hopf actions. One can replace a finite group $G$ acting on an Artin-Schelter algebra $A$ by a semi-simple Hopf algebra $H$ acting on $A$ [Mon2] and study properties of $A^{H}$. We will report some results on this case in [KKZ2]. 
Quotient division algebras. When $A$ is a Noetherian domain and $G$ is a finite group of automorphisms of $A$, then $G$ acts on $Q(A)$, the quotient division ring of $A$. By [Mon1, Theorem 5.3], it is known that $Q(A)^{G}=Q\left(A^{G}\right)$. The classical Noether problem is to determine which linear finite group actions on $k\left[x_{1}, \cdots, x_{n}\right]$ have rational fields of invariants; hence it is a natural question to determine conditions when $Q(A)^{G} \cong Q(A)$. Alev and Dumas have shown that if $G$ is a linear finite abelian group of automorphisms of $D_{n}(\mathbb{C})$, the quotient division algebra of the Weyl algebra $A_{n}(\mathbb{C})$, then $D_{n}(\mathbb{C})^{G} \cong D_{n}(\mathbb{C})$ AD1 (and for any finite group with $n=1$, see [AD2]). One could investigate similar questions for the quotient division algebras of Artin-Schelter regular algebras.

Example 7.1. Let $A$ be the Jordan plane $k_{J}[x, y]$, the algebra generated by $x$ and $y$ with relation $x y-y x=x^{2}$. We have noted that $A$ is rigid, so that $A^{G}$ is never isomorphic to $A$ for any nontrivial finite group of automorphisms. The quotient division algebra is

$$
Q(A)=Q\left(\mathbb{C}\left\langle x, y^{-1}\right\rangle\right)=Q\left(A_{1}(\mathbb{C})\right)=D_{1}(\mathbb{C}) .
$$

Let $G=\langle g\rangle$ be the group of automorphisms generated by the automorphism of $A$ given by $g(x)=-x$ and $g(y)=-y$. Notice that $g$ induces an automorphism of $\mathbb{C}\left\langle x, y^{-1}\right\rangle=A_{1}(\mathbb{C})$, so that by $[\mathrm{AD} 2$,

$$
Q(A)^{G}=Q\left(\mathbb{C}\left\langle x, y^{-1}\right\rangle\right)^{G}=Q\left(A_{1}(\mathbb{C})\right)^{G} \cong D_{1}(\mathbb{C}) .
$$

In this case we have $Q(A)^{G} \cong Q(A)$ even though $A^{G}$ is not isomorphic to $A$.

For $A=k_{-1}[x, y]$ and $g$ the automorphism of $A$ given by $g(x)=-x$ and $g(y)=y$, the invariant subring $A^{G}$ is the commutative polynomial ring $k\left[x^{2}, y\right]$. In this case $Q(A)^{G}=Q\left(A^{G}\right)=k\left(x^{2}, y\right)$ is not isomorphic to $Q(A)$. Unlike the commutative case, our more general notion of reflection groups means that even when $G$ is a reflection group, $Q(A)^{G}$ need not be isomorphic to $Q(A)$.

This paper gives a number of algebras where $A^{G}$ is never isomorphic to $A$, so it would be interesting to determine (a) when $Q(A)^{G}$ is isomorphic to $Q(A)$, and (b) when $Q(A)^{G}$ is isomorphic to $Q(B)$ for an Artin-Schelter regular algebra $B$.

\section{ACKNOWLEDGMENTS}

The authors thank Paul Smith and Ralph Greenberg for several useful discussions and valuable comments. The authors also thank the referee for valuable suggestions. J.J. Zhang was supported by the NSF and the Royalty Research Fund of the University of Washington.

\section{REFERENCES}

[AD1] J. Alev and F. Dumas, Sur les invariants des algèbres de Weyl et de leurs corps de fractions. Rings, Hopf algebras, and Brauer groups (Antwerp/Brussels, 1996), 1-10, Lecture Notes in Pure and Appl. Math., 197, Dekker, New York, 1998. MR1615833 (99g:16042)

[AD2] J. Alev and F. Dumas, Invariants du corps de Weyl sous l'action de groupes finis. Comm. Algebra 25 (1997), no. 5, 1655-1672. MR.1444026 (99c:16030)

[AP] J. Alev and P. Polo, A rigidity theorem for finite group actions on enveloping algebras of semisimple Lie algebras, Adv. Math. 111 (1995), no. 2, 208-226. MR.1318528(95m:16015)

[AS] M. Artin and W. F. Schelter, Graded algebras of global dimension 3, Adv. in Math. 66 (1987), no. 2, 171-216. MR917738 (88k:16003)

[ATV1] M. Artin, J. Tate and M. Van den Bergh, Some algebras associated to automorphisms of elliptic curves, "The Grothendieck Festschrift," Vol. I, ed. P. Cartier et al., Birkhäuser Boston, 1990, 33-85. MR1086882 (92e:14002) 
[ATV2] M. Artin, J. Tate and M. Van den Bergh, Modules over regular algebras of dimension 3, Invent. Math. 106 (1991), no. 2, 335-388. MR.1128218 (93e:16055)

[AZ] M. Artin and J. J. Zhang, Noncommutative projective schemes, Adv. Math. 109 (1994), 228-287. MR1304753 (96a:14004)

[BR] G. Benkart and T. Roby, Down-up algebras, J. Algebra 209 (1998), 305-344. Addendum, J. Algebra 213 (1999), no. 1, 378. MR.1652138 (2000e:06001a); MR1674692 (2000e:06001b)

[Be] D.J. Benson, Polynomial invariants of finite groups, London Mathematical Society Lecture Note Series, 190. Cambridge University Press, Cambridge, 1993. MR1249931 $(94 \mathrm{j}: 13003)$

[CE] A. Clark and J. Ewing, The realization of polynomial algebras as cohomology rings, Pacific J. Math. 50 (1974), 425-434. MR0367979(51:4221)

[Co] H.S.M. Coxeter, Discrete groups generated by reflections, Ann. of Math. (2) 35 (1934), no. 3, 588-621. MR 1503182

[Go] N.L. Gordeev, Invariants of linear groups generated by matrices with two nonidentity eigenvalues, Zap. Nauchn. Sem. Leningrad. Otdel. Mat. Inst. Steklov. (LOMI) 114 (1982), 120-130; English translation in J. Soviet Math. 27 (1984), no. 4. MR669563 (83m:20067)

[HW] G. H. Hardy and E. M Wright, "An Introduction to the Theory of Numbers", Fourth Edition, Oxford University Press, London, 1960. MR0067125 (16:673c)

[JiZ] N. Jing and J.J. Zhang, On the trace of graded automorphisms, J. Algebra 189 (1997), no. 2, 353-376. MR1438180 (98f:16029)

[JoZ] P. Jørgensen and J.J. Zhang, Gourmet's guide to Gorensteinness, Adv. Math. 151 (2000), no. 2, 313-345. MR1758250 (2001d:16023)

[Jos] A. Joseph, Coxeter structure and finite group action, Algèbre non commutative, groupes quantiques et invariants (Reims, 1995), 185-219, Sémin. Congr., 2, Soc. Math. France, Paris, 1997. MR1601143 (98k:17011)

$[\mathrm{KW}] \quad$ V. Kac and K. Watanabe, Finite linear groups whose ring of invariants is a complete intersection, Bull, Amer. Math. Soc. (N.S.) 6 (1982), no. 2, 221-223. MR640951 (83h:14042)

[KKZ1] E. Kirkman, J. Kuzmanovich and J.J. Zhang, A Shephard-Todd-Chevalley theorem for noncommutative regular algebras, in preparation.

[KKZ2] E. Kirkman, J. Kuzmanovich and J.J. Zhang, Hopf algebra (co)-actions on Artin-Schelter regular algebras, in preparation.

[KMP] E. Kirkman, I. Musson and D. Passman, Noetherian down-up algebras, Proc. Amer. Math. Soc. 127 (1999), 3161-3167. MR:1610796 (2000b:16042)

[KL] G. Krause and T. Lenagan, "Growth of Algebras and Gelfand-Kirillov Dimension, revised edition", Graduate Studies in Mathematics, Vol. 22, AMS, Providence, 2000. MR1721834 (2000j:16035)

[Le] T. Levasseur, Some properties of noncommutative regular rings, Glasgow Math. J. 34 (1992), 277-300. MR 1181768 (93k:16045)

[MR] J. C. McConnell and J. C . Robson, "Noncommutative Noetherian Rings," Wiley, Chichester, 1987. MR934572 (89j:16023)

[Mo] R. Mollin, "Algebraic Number Theory". Chapman \& Hall/CRC, Boca Raton, FL, 1999. MR $1682930(2000 \mathrm{e}: 11130)$

[Mon1] S. Montgomery, "Fixed rings of finite automorphism groups of associative rings", Lecture Notes in Mathematics, 818. Springer, Berlin, 1980 MR.590245 (81j:16041)

[Mon2] S. Montgomery, "Hopf algebras and their actions on rings", CBMS Regional Conference Series in Mathematics, 82, Providence, RI, 1993. MR1243637 (94i:16019)

[RRZ] Z. Reichstein, D. Rogalski and J.J. Zhang, Projectively simple rings, Adv. in Math, 203 (2006), 365-407. MR:2227726 (2007i:16071)

[ShT] G.C. Shephard and J.A. Todd, Finite unitary reflection groups, Canadian J. Math. 6, (1954). 274-304. MR0059914 (15:600b)

[Sm1] S.P. Smith, Can the Weyl algebra be a fixed ring?, Proc. Amer. Math. Soc. 107 (1989), no. 3, 587-589. MR962247 (90b:16010)

[Sm2] S.P. Smith, Some finite-dimensional algebras related to elliptic curves, Representation theory of algebras and related topics (Mexico City, 1994), 315-348, CMS Conf. Proc., 19, AMS, Providence, RI, 1996. MR1388568 (97e:16053)

[SmS] S. P. Smith and J. T. Stafford, Regularity of the four-dimensional Sklyanin algebra, Compositio Math. 83 (1992), no. 3, 259-289. MR1175941 (93h:16037) 
[StZ] D.R. Stephenson and J.J. Zhang, Growth of graded Noetherian rings, Proc. Amer. Math. Soc. 125 (1997), no. 6, 1593-1605. MR1371143 (97g:16033)

[TV] J. Tate and M. Van den Bergh, Homological properties of Sklyanin algebras, Invent. Math. 124 (1996), no. 1-3, 619-647. MR.1369430 (98c:16057)

[Zh] J.J. Zhang, Connected graded Gorenstein algebras with enough normal elements, J. Algebra 189 (1997), no. 2, 390-405. MR1438182(97m:16018)

Department of Mathematics, Wake Forest University, P.O. Box 7388, Winston-Salem, North Carolina 27109

E-mail address: kirkman@wfu.edu

Department of Mathematics, Wake Forest University, P.O. Box 7388, Winston-Salem, North Carolina 27109

E-mail address: kuz@wfu.edu

Department of Mathematics, University of Washington, Box 354350, Seattle, WashINGTON 98195

E-mail address: zhang@math.washington.edu 